\title{
On the Analyticity of Laguerre Series
}

\author{
Ernst Joachim Weniger \\ Institut für Physikalische und Theoretische Chemie \\ Universität Regensburg, D-93040 Regensburg, Germany \\ To appear in Journal of Physics A \\ Accepted: 27 August 2008
}

\begin{abstract}
The transformation of a Laguerre series $f(z)=\sum_{n=0}^{\infty} \lambda_{n}^{(\alpha)} L_{n}^{(\alpha)}(z)$ to a power series $f(z)=\sum_{n=0}^{\infty} \gamma_{n} z^{n}$ is discussed. Since many nonanalytic functions can be expanded in terms of generalized Laguerre polynomials, success is not guaranteed and such a transformation can easily lead to a mathematically meaningless expansion containing power series coefficients that are infinite in magnitude. Simple sufficient conditions based on the decay rates and sign patters of the Laguerre series coefficients $\lambda_{n}^{(\alpha)}$ as $n \rightarrow \infty$ can be formulated which guarantee that the resulting power series represents an analytic function. The transformation produces a mathematically meaningful result if the coefficients $\lambda_{n}^{(\alpha)}$ either decay exponentially or factorially as $n \rightarrow \infty$. The situation is much more complicated but also much more interesting - if the $\lambda_{n}^{(\alpha)}$ decay only algebraically as $n \rightarrow \infty$. If the $\lambda_{n}^{(\alpha)}$ ultimately have the same sign, the series expansions for the power series coefficients diverge, and the corresponding function is not analytic at the origin. If the $\lambda_{n}^{(\alpha)}$ ultimately have strictly alternating signs, the series expansions for the power series coefficients still diverge, but are summable to something finite, and the resulting power series represents an analytic function. If algebraically decaying and ultimately alternating Laguerre series coefficients $\lambda_{n}^{(\alpha)}$ possess sufficiently simple explicit analytical expressions, the summation of the divergent series for the power series coefficients can often be accomplished with the help of analytic continuation formulas for hypergeometric series ${ }_{p+1} F_{p}$, but if the $\lambda_{n}^{(\alpha)}$ have a complicated structure or if only their numerical values are available, numerical summation techniques have to be employed. It is shown that certain nonlinear sequence transformations - in particular the so-called delta transformation [E. J. Weniger, Comput. Phys. Rep. 10, 189 - 371 (1989), Eq. (8.4-4)] - are able to sum the divergent series occurring in this context effectively. As a physical application of the results of this article, the legitimacy of the rearrangement of certain one-range addition theorems for Slater-type functions [I. I. Guseinov, Phy. Rev. A 22, 369 - 371 (1980); Int. J. Quantum Chem. 81, 126 - 129 (2001); Int. J. Quantum Chem. 90, 114 - 118 (2002)] is investigated.
\end{abstract}

PACS numbers: 02.30.Gp, 02.30.Lt, 02.30.Mv, 02.60.-x

AMS classification scheme numbers: 30B10, 30B40, 33C45, 40A05, 65B10

Keywords: Generalized Laguerre polynomials, Laguerre series, analyticity, divergent series, summability, nonlinear sequence transformations 


\section{Contents}

1 Introduction 3

2 Generalized Laguerre Polynomials 5

3 The Laguerre Series for the General Power Function 8

4 Algebraically Decaying Series Coefficients 11

5 Exponentially and Factorially Decaying Series Coefficients 16

6 Computational Approaches $\quad 20$

7 Guseinov's Rearranged One-Range Addition Theorems 26

8 Summary and Conclusions

A Divergent Series $\quad 33$

B Sequence Transformations $\quad 34$

Bibliography 


\section{Introduction}

The generalized Laguerre polynomials $L_{n}^{(\alpha)}(z)$, whose most relevant properties are reviewed in Section 2] are a very important class of orthogonal polynomials with numerous mathematical and physical applications. There is an extensive literature both on their mathematical properties as well as on their applications, and any attempt of providing a reasonably complete bibliography would be hopeless. Let me just mention that the radial parts of bound state hydrogen eigenfunctions and of several other physically relevant complete and orthonormal sets of function $f: \mathbb{R}^{3} \rightarrow \mathbb{C}$ are essentially generalized Laguerre polynomials (see for example [135. Sections IV and V] and references therein).

As discussed in more detail in Section 2, the generalized Laguerre polynomials $L_{n}^{(\alpha)}(z)$ form a complete orthogonal polynomial system in the weighted Hilbert space $L_{z^{\alpha} \mathrm{e}^{-z}}^{2}([0, \infty))$ defined by (2.7), which is based on an integration over the interval $[0, \infty)$ involving the weight function $w(z)=z^{\alpha} \exp (-z)$. Accordingly, the topic of this article are infinite Laguerre series of the following type:

$$
\begin{aligned}
& f(z)=\sum_{n=0}^{\infty} \lambda_{n}^{(\alpha)} L_{n}^{(\alpha)}(z) \\
& \lambda_{n}^{(\alpha)}=\frac{n !}{\Gamma(\alpha+n+1)} \int_{0}^{\infty} z^{\alpha} \mathrm{e}^{-z} L_{n}^{(\alpha)}(z) f(z) \mathrm{d} z
\end{aligned}
$$

The expansion coefficients $\lambda_{n}^{(\alpha)}$ are essentially inner products, utilizing the orthogonality and the completeness of the generalized Laguerre polynomials in $L_{z^{\alpha} \mathrm{e}^{-z}}^{2}([0, \infty))$.

Not all expansions in terms of generalized Laguerre polynomials can be interpreted in a Hilbert space setting. There are expansions of special functions in terms of generalized Laguerre polynomials with variable and index-dependent superscripts. A simple example is the generating function [102, p. 242]

$$
\sum_{n=0}^{\infty} L_{n}^{(\alpha-n)}(x) t^{n}=\mathrm{e}^{-x t}(1+t)^{\alpha}, \quad|t|<1 .
$$

Expansions of that kind cannot be derived via a straightforward application of the orthogonality of the generalized Laguerre polynomials. In the article by Sánchez-Ruiz, López-Artéz, and Dehesa [113], transformation formulas for generalized hypergeometric series were employed for the construction of Laguerre expansions with variable superscripts. However, nonorthogonal Laguerre expansions of that kind are not the topic of this article.

It is generally accepted that orthogonal expansions are extremely useful mathematical tools and that they have many highly advantageous features. This is, however, not the whole truth, in particular if we want to approximate functions. There are situations, in which alternative representations are (much) more useful. Hilbert space theory only guarantees that an orthogonal expansion converges in the mean with respect to the corresponding norm, but not necessarily pointwise or even uniformly. Therefore, orthogonal expansions are not necessarily the best choice if we are interested in local properties of functions.

Convergence in the mean is - although completely satisfactory for many purposes such as the evaluation of integrals - a comparatively weak form of convergence. In practice, it is therefore often desirable or even necessary to construct alternative representations possessing more convenient properties (see also the discussion in [46]).

A very desirable feature of functions $f: \mathbb{C} \rightarrow \mathbb{C}$ is analyticity in the sense of complex analysis. This means that a function $f$ can be represented in a neighborhood of the origin by 
a convergent power series,

$$
f(z)=\sum_{n=0}^{\infty} \gamma_{n} z^{n}
$$

and that the coefficients $\gamma_{n}$ of this power series essentially correspond to the derivatives of $f$ at $z=0$.

If the function $f$ defined by the Laguerre series 11.1 is explicitly known, it is usually not too difficult to decide whether $f$ is analytic or not, and if $f$ is analytic it is normally not too difficult to construct at least the leading terms of its power series expansion (1.3).

Unfortunately, the situation is not always so good, and it can even happen that only the numerical values of a finite number of expansion coefficients $\lambda_{n}^{(\alpha)}$ in 1.1 are known. In such a case, it would certainly be helpful if we could relate the properties of the expansion coefficients $\lambda_{n}^{(\alpha)}$ - in particular their decay rate and their sign pattern - to the analyticity of the function $f(z)$ defined by the Laguerre series (1.1). It would also be helpful if we could construct at least the leading power series coefficients $\gamma_{n}$ - either exactly or in an approximate sense - from the coefficients $\lambda_{n}^{(\alpha)}$ of the Laguerre series (1.1). .

Pollard [107], Szász and Yeardley [125], and Rusev [111] had investigated Laguerre expansions of the type of (1.1) of analytic functions and analyzed their regions of convergence. However, the inverse problem - the formulation of criteria which guarantee that a Laguerre expansion of the type of (1.1) represents a function analytic in a neighborhood of the origin seems to be essentially unexplored. I am only aware of short remarks by Gottlieb and Orszag [49, p. 42] and by Doha [41, p. 5452], respectively, who stated without detailed proof that such a Laguerre series converges faster than algebraically if the function under consideration is analytic at the origin.

Many Laguerre series are known which seem to confirm the claim of Gottlieb and Orszag and of Doha, respectively. The probably most simple example is the well known generating function $(1-t)^{-\alpha-1} \exp (z t /[t-1])$ of the Laguerre polynomials. For $|t|<1$, it is an entire function, and the coefficients of its Laguerre series (5.8) decay exponentially for $|t|<1$.

Another example is the Laguerre series (3.3) for the general power function $z^{\rho}$ with nonintegral $\rho \in \mathbb{R} \backslash \mathbb{N}_{0}$. Obviously, $z^{\rho}$ with $\rho \in \mathbb{R} \backslash \mathbb{N}_{0}$ is not analytic at $z=0$. The series coefficients in (3.3) decay algebraically and possess for sufficiently large indices the same sign. As shown in Section 3, it is nevertheless possible to transform the Laguerre series 3.3 for $z^{\rho}$ with $\rho \in \mathbb{R} \backslash \mathbb{N}_{0}$ to the formal power series (3.7). However, the formal power series 3.7 is not a mathematically meaningful object: For sufficiently large indices, the power series coefficients in (3.7) are according to (3.9) all infinite in magnitude. Thus, the Laguerre series (3.3) for $z^{\rho}$ with $\rho \in \mathbb{R} \backslash \mathbb{N}_{0}$ also agrees with the claim of Gottlieb and Orszag and of Doha, respectively.

If, however, we augment the Laguerre series (3.3) for $z^{\rho}$ by an additional alternating sign $(-1)^{n}$, we obtain the Laguerre series (4.7). Its transformation to a power series yields according to (4.12) a confluent hypergeometric function ${ }_{1} F_{1}(-\rho ; \alpha+1 ; z / 2)$, which for $\operatorname{Re}(\alpha)>-1$ is an entire function. The coefficients in the Laguerre series 4.7) decay algebraically in magnitude. This implies that the transformation of (4.7) to a power series leads to power series coefficients $\gamma_{n}$ that are represented by divergent series expansions. However, the alternating $\operatorname{sign}(-1)^{n}$ in (4.7) makes it possible to associate finite values to these divergent series, i.e., the series expansions for the power series coefficients are now summable. Thus, we obtain a function, which is analytic at $z=0$ and which does not agree with the claim of Gottlieb and Orszag and of Doha, respectively.

The example of the closely related Laguerre series (3.3) and 4.7) shows that it makes a huge difference if Laguerre series coefficients $\lambda_{n}^{(\alpha)}$, that decay algebraically in magnitude, ultimately have strictly alternating or strictly monotone signs. Thus, the claim of Gottlieb and

Ernst Joachim Weniger: On the Analyticity of Laguerre Series 
Orszag and of Doha, respectively, is imprecise and ignores the pivotal role of divergent, but summable series expansions for the power series coefficients $\gamma_{n}$ in (1.3). Some basic facts about the summation of divergent series are reviewed in Appendix A

A detailed investigation of the analyticity of functions represented by Laguerre expansions of the type of (1.1) is the topic of this article. The central result is the transformation formula (3.14), which yields a formal power series expansion for a function represented by a Laguerre expansion. The crucial question is whether the inner $\mu$ series in (3.14) for the power series coefficients $\gamma_{n}$ converge. If these $\mu$ series do not converge and are also not summable to something finite, then the function under consideration is not analytic at the origin and the resulting formal power series that is mathematically meaningless since contains coefficients $\gamma_{n}$ which are infinite in magnitude. Consequently, it is comparatively easy to relate the analyticity of a function $f$ represented by a Laguerre series at the origin $z=0$ with the decay rate and the sign pattern of its coefficients $\lambda_{n}^{(\alpha)}$.

Analytical manipulations can only lead to closed form expressions for the power series coefficients $\gamma_{n}$ if the Laguerre series coefficients $\lambda_{n}^{(\alpha)}$ possess a sufficiently simple structure. This undeniable fact may create the false impression that the formalism developed in this article is restricted to Laguerre series with very simple coefficients $\lambda_{n}^{(\alpha)}$, and that this formalism is at best suited for an alternative rederivation of known generating functions of the generalized Laguerre polynomials.

As discussed in Section 6 it is, however, often possible to construct numerical approximations to the leading power series coefficients $\gamma_{n}$ from the coefficients $\lambda_{n}^{(\alpha)}$ of the Laguerre series, even if the inner $\mu$ series in (3.14) diverges. The necessary summations can be done effectively with the help of certain nonlinear sequence transformations - Wynn's epsilon algorithm (B.4) and the two Levin-type transformations (B.14) and (B.15) - whose basic properties are reviewed in Appendix B. While Wynn's epsilon algorithm (B.4), which produces Padé approximants if the input data are the partial sums of a power series, is now fairly well known among (applied) mathematicians and theoretical physicists, the Levin-type transformations (B.14) and (B.15), which in some cases were found to be remarkably powerful, are not nearly as well known as they deserve to be.

As a physical application of the mathematical formalism developed in this article, the legitimacy of rearrangements of certain one-range addition theorems for Slater-type functions with in general nonintegral principal quantum numbers performed by Guseinov [53, 54, 56] is investigated in Section 7 It is shown that the one-center limits of Guseinov's rearranged addition theorems do not exist if the principal quantum numbers of the Slater-type functions are nonintegral.

\section{Generalized Laguerre Polynomials}

The generalized Laguerre polynomials $L_{n}^{(\alpha)}(z)$ with $n \in \mathbb{N}_{0}$ and $\operatorname{Re}(\alpha)>-1$ are orthogonal polynomials associated with the integration interval $[0, \infty)$ and the weight function $w(z)=$ $z^{\alpha} \exp (-z)$.

In this article, the mathematical notation for generalized Laguerre polynomials (see for example [102, Chapter 5.5]) is used. Additional conventions, as used predominantly in older books and articles on quantum theory, were discussed by Kaijser and Smith [87, Footnote 1 on p. 48] and also in [151, Section 4].

The generalized Laguerre polynomials possess an explicit expression as a terminating

Ernst Joachim Weniger: On the Analyticity of Laguerre Series 
confluent hypergeometric series [102. p. 240],

$$
L_{n}^{(\alpha)}(z)=\frac{(\alpha+1)_{n}}{n !}{ }_{1} F_{1}(-n ; \alpha+1 ; z),
$$

and they can also be defined via their Rodrigues relationship [102, p. 241]

$$
L_{n}^{(\alpha)}(z)=z^{-\alpha} \frac{\mathrm{e}^{z}}{n !} \frac{\mathrm{d}^{n}}{\mathrm{~d} z^{n}}\left[\mathrm{e}^{-z} z^{n+\alpha}\right] .
$$

In the special case $\alpha=0$, it is common to drop the superscript since we obtain the usual Laguerre polynomials [102, p. 239]:

$$
L_{n}(z)=L_{n}^{(0)}(z), \quad n \in \mathbb{N}_{0} .
$$

Either the explicit expression (2.1) or the Rodrigues relationship (2.2) can be used to define generalized Laguerre polynomials with essentially arbitrary complex values of the superscript $\alpha$. But in the orthogonality relationship of the generalized Laguerre polynomials [102, $\mathrm{p}$. 241],

$$
\int_{0}^{\infty} z^{\alpha} \mathrm{e}^{-z} L_{m}^{(\alpha)}(z) L_{n}^{(\alpha)}(z) \mathrm{d} z=\frac{\Gamma(\alpha+n+1)}{n !} \delta_{m n}, \quad m, n \in \mathbb{N}_{0},
$$

it is necessary to assume $\operatorname{Re}(\alpha)>-1$ because otherwise this integral does not exist. However, as discussed by Kochneff [95], this condition can be relaxed if this and related integrals are reinterpreted as Hadamard finite part integrals.

In the vast majority of calculations involving generalized Laguerre polynomials, the superscript $\alpha$ is real and also positive. In practice, more general complex values of $\alpha$ play a negligible rule. Therefore, in the following text it is always tacitly assumed that all integrals exist in the ordinary sense, which avoids the complications of Hadamard finite part integrals, and that the the superscript $\alpha$ of a generalized Laguerre polynomial is a real number satisfying $\alpha>-1$.

The orthogonality relationship (2.4) suggests the introduction of the following inner product containing the weight function $w(z)=z^{\alpha} \exp (-z)$ for functions $f, g: \mathbb{C} \rightarrow \mathbb{C}$ :

$$
(f \mid g)_{z^{\alpha} \mathrm{e}^{-z, 2}}=\int_{0}^{\infty} z^{\alpha} \mathrm{e}^{-z}[f(z)]^{*} g(z) \mathrm{d} z
$$

This inner product gives rise to the norm

$$
\|f\|_{z^{\alpha} \mathrm{e}^{-z, 2}}=\sqrt{(f \mid f)_{z^{\alpha} \mathrm{e}^{-z, 2}}} .
$$

We then obtain the following Hilbert space of square integrable functions:

$$
\begin{aligned}
L_{z^{\alpha} \mathrm{e}^{-z}}^{2}([0, \infty)) & =\left\{f:\left.\mathbb{C} \rightarrow \mathbb{C}\left|\int_{0}^{\infty} z^{\alpha} \mathrm{e}^{-z}\right| f(z)\right|^{2} \mathrm{~d} z<\infty\right\} \\
& =\left\{f: \mathbb{C} \rightarrow \mathbb{C} \mid\|f\|_{z^{\alpha} \mathrm{e}^{-z}, 2}<\infty\right\} .
\end{aligned}
$$

The completeness of the generalized Laguerre polynomials in this weighted Hilbert space is a classic result of mathematical analysis (see for example [79, p. 33], [114, pp. 349 - 351], or [130, pp. 235 - 238]). Thus, the normalized polynomials

$$
\mathscr{L}_{n}^{(\alpha)}(z)=\left[\frac{n !}{\Gamma(\alpha+n+1)}\right]^{1 / 2} L_{n}^{(\alpha)}(z), \quad n \in \mathbb{N}_{0}
$$


are a complete and orthonormal polynomial system in $L_{z^{\alpha} \mathrm{e}^{-z}}^{2}([0, \infty))$, satisfying

$$
\int_{0}^{\infty} z^{\alpha} \mathrm{e}^{-z} \mathscr{L}_{m}^{(\alpha)}(z) \mathscr{L}_{n}^{(\alpha)}(z) \mathrm{d} z=\delta_{m n}
$$

Accordingly, every $f \in L_{z^{\alpha} \mathrm{e}^{-z}}^{2}([0, \infty))$ can be expanded in terms of the normalized polynomials $\left\{\mathscr{L}_{n}^{(\alpha)}(z)\right\}_{n=0}^{\infty}$ :

$$
\begin{aligned}
f(z) & =\sum_{n=0}^{\infty} \mathscr{C}_{n}^{(\alpha)} \mathscr{L}_{n}^{(\alpha)}(z) \\
\mathscr{C}_{n}^{(\alpha)} & =\int_{0}^{\infty} z^{\alpha} \mathrm{e}^{-z} \mathscr{L}_{n}^{(\alpha)}(z) f(z) \mathrm{d} z .
\end{aligned}
$$

This expansion in terms of the normaized polynomials $\mathscr{L}_{n}^{(\alpha)}(z)$, which converges in the mean with respect to the norm (2.6), is nothing but the Laguerre series (1.1) in disguise. In agreement with 2.8), we only have to choose

$$
\mathscr{C}_{n}^{(\alpha)}=\left[\frac{\Gamma(\alpha+n+1)}{n !}\right]^{1 / 2} \lambda_{n}^{(\alpha)}
$$

to see that the expansions 1.1 and 2.10 are identical.

Let me emphasize once more that Laguerre expansions converge in general only in the mean, but not necessarily pointwise (see for example [2]). Additional conditions, which a function has to satisfy in order to guarantee that its Laguerre expansion also converges pointwise, were discussed by Szegö [126, Theorem 9.1.5 on p. 246].

Hilbert space theory can be used to derive sufficient criteria, which the coefficients $\lambda_{n}^{\alpha}$ or $\mathscr{C}_{n}^{(\alpha)}$ have to satisfy in order to guarantee that the equivalent expansions (1.1) and (2.10) converge in the mean. The basic requirement is that the norm (2.6) of both $f$ and its Laguerre expansion (2.10) must be finite:

$$
\|f\|_{z^{\alpha} \mathrm{e}^{-z, 2}}=\left\{\sum_{n=0}^{\infty}\left|\mathscr{C}_{n}^{(\alpha)}\right|^{2}\right\}^{1 / 2}<\infty .
$$

It follows from 2.11) that this condition can also be reformulated as follows:

$$
\|f\|_{z^{\alpha} \mathrm{e}^{-z, 2}}=\left\{\sum_{n=0}^{\infty} \frac{\Gamma(\alpha+n+1)}{n !}\left|\lambda_{n}^{(\alpha)}\right|^{2}\right\}^{1 / 2}<\infty .
$$

A sufficient condition, which guarantees that the infinite series $\sum_{n=0}^{\infty}\left|\mathscr{C}_{n}^{(\alpha)}\right|^{2}$ in 2.12 converges, is

$$
\left|\mathscr{C}_{n}^{(\alpha)}\right|^{2} \sim n^{-1-\varepsilon}, \quad n \rightarrow \infty, \quad \varepsilon>0 .
$$

It follows from 2.11) and 2.14) that the Laguerre series (1.1) converges in the mean if its coefficients $\lambda_{n}^{(\alpha)}$ satisfy

$$
\frac{\Gamma(\alpha+n+1)}{n !}\left|\lambda_{n}^{(\alpha)}\right|^{2} \sim n^{-1-\varepsilon}, \quad n \rightarrow \infty, \quad \varepsilon>0 .
$$

Now, we need the asymptotic approximation [1, Eq. (6.1.47) on p. 257]

$$
\Gamma(z+a) / \Gamma(z+b)=z^{a-b}[1+\mathrm{O}(1 / z)], \quad z \rightarrow \infty,
$$


which is the leading term of an asymptotic expansion first derived by Tricomi and Erdélyi [131] that holds - as emphasized by Olver [104, p. 119] - without restrictions on $a, b \in \mathbb{C}$. Thus, we obtain:

$$
\frac{\Gamma(\alpha+n+1)}{n !} \sim n^{\alpha}+\mathrm{O}\left(n^{\alpha-1}\right), \quad n \rightarrow \infty
$$

This translates to the sufficient convergence condition that $\left|\lambda_{n}^{(\alpha)}\right|$ must decay like

$$
\left|\lambda_{n}^{(\alpha)}\right| \sim n^{-[\alpha+\varepsilon+1] / 2}, \quad n \rightarrow \infty, \quad \varepsilon>0
$$

or faster. If this asymptotic condition is satisfied, then the Laguerre series (1.1) for $f(z)$ converges in the mean with respect to the norm (2.6) of the Hilbert space $L_{\mathrm{e}^{-z} z^{\alpha}}^{2}([0, \infty))$.

The sufficient convergence condition (2.18) shows that the coefficients $\lambda_{n}^{(\alpha)}$ can decay extremely slowly, which translates to a possibly extremely slow convergence of Laguerre series of the type of (1.1). Thus, it would be overly optimistic to assume that all Laguerre series are necessarily computationally useful.

\section{The Laguerre Series for the General Power Function}

In the theory of orthogonal expansions, which converge in the mean with respect to the norm $\|\cdot\|$ of some Hilbert space $\mathscr{H}$, the decisive criterion is that the function, which is to be expanded, has to belong to $\mathscr{H}$. Consequently, any function $f: \mathbb{C} \rightarrow \mathbb{C}$ with finite norm (2.6) belongs to the weighted Hilbert space $L_{z \alpha}^{2} \mathrm{e}^{-z}([0, \infty))$ and can be expanded in terms of generalized Laguerre polynomials according to (1.1). The resulting expansion (1.1) converges in the mean with respect to the norm (2.6) of the Hilbert space $L_{z^{\alpha} \mathrm{e}^{-z}}^{2}([0, \infty))$.

The existence and convergence of a Laguerre series of the type of (1.1) does not allow any conclusion about the analyticity of the corresponding function. The weighted Hilbert space $L_{z^{\alpha} \mathrm{e}^{-z}}^{2}([0, \infty))$ contains many functions that are obviously not analytic at the origin. Therefore, attempts of constructing a power series from the Laguerre series can easily lead to a disaster. The complications, which can occur in this context, can be demonstrated via the general power function $z^{\rho}$ with nonintegral $\rho \in \mathbb{R} \backslash \mathbb{N}_{0}$.

In integrals over the positive real semi-axis, the weight function $z^{\alpha} \mathrm{e}^{-z}$ becomes at least for $\operatorname{Re}(\alpha)>0$ very small both as $z \rightarrow 0$ and as $z \rightarrow \infty$. Consequently, $z^{\alpha} \mathrm{e}^{-z}$ suppresses in the inner product (2.5) the contribution of the remaining integrand for small and large arguments. Therefore, the general power function $z^{\rho}$ with $\rho \in \mathbb{R} \backslash \mathbb{N}_{0}$ possesses at least for sufficiently large values of $\alpha$ an expansion of the type of (1.1) in terms of generalized Laguerre polynomials. The existence of this expansion is guaranteed if $z^{\rho}$ belongs to the Hilbert space $L_{z^{\alpha} \mathrm{e}^{-z}}^{2}([0, \infty))$, or equivalently, if

$$
\left|\int_{0}^{\infty} z^{\alpha+2 \rho} \mathrm{e}^{-z} \mathrm{~d} z\right|<\infty
$$

holds. Thus, we have to require that $\alpha+2 \rho>-1$ holds.

For the construction of a Laguerre series for $z^{\rho}$, we only need the integral [50, Eq. 
(7.414.7) on p. 850]

$$
\begin{aligned}
\int_{0}^{\infty} \mathrm{e}^{-s t} t^{\beta} L_{n}^{(\alpha)}(t) \mathrm{d} t & \\
= & \frac{\Gamma(\beta+1) \Gamma(\alpha+n+1)}{n ! \Gamma(\alpha+1)} s^{-\beta-1}{ }_{2} F_{1}(-n, \beta+1 ; \alpha+1 ; 1 / s), \\
& \operatorname{Re}(\beta)>-1, \quad \operatorname{Re}(s)>0,
\end{aligned}
$$

to obtain after some essentially straightforward algebra [43, Eq. (16) on p. 214]:

$$
z^{\rho}=\frac{\Gamma(\rho+\alpha+1)}{\Gamma(\alpha+1)} \sum_{n=0}^{\infty} \frac{(-\rho)_{n}}{(\alpha+1)_{n}} L_{n}^{(\alpha)}(z), \quad \rho \in \mathbb{R} \backslash \mathbb{N}_{0}, \quad \alpha+2 \rho>-1 .
$$

If we set $\rho=m$ with $m \in \mathbb{N}_{0}$, then the infinite series on the right-hand side terminates because of the Pochhammer symbol $(-m)_{n}$ and we obtain the following finite sum (see for example [109, Eq. (2) on p. 207]):

$$
z^{m}=(\alpha+1)_{m} \sum_{n=0}^{m} \frac{(-m)_{n}}{(\alpha+1)_{n}} L_{n}^{(\alpha)}(z), \quad m \in \mathbb{N}_{0}, \quad \alpha+2 m>-1 .
$$

Although intimately related, there are nevertheless some fundamental differences between the two Laguerre expansions (3.3) and (3.4). The finite sum formula (3.4) is a relationship among polynomials and therefore certainly valid pointwise for arbitrary $z \in \mathbb{C}$ as well as analytic at the origin in the sense of complex analysis.

In the case of the infinite series expansion (3.3), we only know that it converges in the mean with respect to the norm (2.6), but we have no a priori reason to assume that this expansion might converge pointwise for arbitrary $\rho \in \mathbb{R} \backslash \mathbb{N}_{0}$. Moreover, $z^{\rho}$ with $\rho \in \mathbb{R} \backslash \mathbb{N}_{0}$ is not analytic at the origin.

The validity of the finite sum (3.4) can be checked by inserting the explicit expression (2.1) for the generalized Laguerre polynomials. If we then rearrange the order of the two nested sums, we obtain after some algebra:

$$
z^{m}=(\alpha+1)_{m} \sum_{k=0}^{m}(-1)^{k} \frac{(-m)_{k}}{(\alpha+1)_{k}} \frac{z^{k}}{k !} \sum_{v=0}^{m-k}(-1)^{v}\left(\begin{array}{c}
m-k \\
v
\end{array}\right)
$$

Next, we use the relationship [1, Eq. (3.1.7) on p. 10]:

$$
\sum_{k=0}^{n}(-1)^{k}\left(\begin{array}{l}
n \\
k
\end{array}\right)=\delta_{n 0}, \quad n \in \mathbb{N}_{0},
$$

for binomial sums, which shows that the inner sum in (3.5) vanishes unless we have $k=m$. Thus, we only need $(-1)^{m}(-m)_{m}=(1)_{m}=m$ ! to arrive at the trivial identity $z^{m}=z^{m}$ which proves the correctness of (3.4).

In the case of the infinite series $(3.3)$ for $z^{\rho}$ with $\rho \in \mathbb{R} \backslash \mathbb{N}_{0}$, we can also insert the explicit expression (2.1) for the generalized Laguerre polynomials into it and rearrange the order of summations. We then obtain after some algebra:

$$
z^{\rho}=\frac{\Gamma(\rho+\alpha+1)}{\Gamma(\alpha+1)} \sum_{k=0}^{\infty}(-1)^{k} \frac{(-\rho)_{k}}{(\alpha+1)_{k}} \frac{z^{k}}{k !}{ }_{1} F_{0}(k-\rho ; 1) .
$$


Superficially, it looks as if we succeeded in constructing a power series expansion for the in general nonintegral power $z^{\rho}$. However, the generalized hypergeometric series ${ }_{1} F_{0}$ with unit argument is the limiting case $z \rightarrow 1$ of the so-called binomial series [102, p. 38]:

$$
{ }_{1} F_{0}(a ; z)=\sum_{m=0}^{\infty}\left(\begin{array}{c}
-a \\
m
\end{array}\right)(-z)^{m}=(1-z)^{-a}, \quad|z|<1 .
$$

If we set $a=k-\rho$ with $k \in \mathbb{N}_{0}$ and $\rho \in \mathbb{R} \backslash \mathbb{N}_{0}$, we obtain for the hypergeometric series ${ }_{1} F_{0}$ in (3.7):

$$
{ }_{1} F_{0}(k-\rho ; 1)=\lim _{z \rightarrow 1}(1-z)^{\rho-k}= \begin{cases}\infty, & \rho<0, \\ 0, & k<\rho \geq 0, \\ \infty, & k>\rho \geq 0 .\end{cases}
$$

Thus, disaster struck and the power series 3.7 contains infinitely many series coefficients that are infinite in magnitude.

I am aware of an article by Villani [133] who tried to make sense of perturbation expansions with divergent terms. Since, however, this is the only article on this topic, which I am aware of, I am tempted to believe that Villani's approach was not overly fertile. Therefore, I will stick to the usual mathematical convention that a power series with coefficients, that are infinite in magnitude, does not exist as a mathematically meaningful object.

It is important to note that series with divergent terms and divergent series are not the same. In the case of divergent series, all terms are finite, but the conventional process of adding up the terms successively does not lead to a convergent result. Nevertheless, it is often possible to associate a finite value to a divergent series with the help of a suitable summation technique. As reviewed in Appendix A, divergent series and their summation have been and to some extent still are a highly controversial topic. The summation of divergent series plays a major role in Sections (4) and (6).

Since the hypergeometric series ${ }_{1} F_{0}(k-\rho ; 1)$ in 3.7 does not converge for all indices $k$, the Laguerre series (3.3) for $z^{\rho}$ with $\rho \in \mathbb{R} \backslash \mathbb{N}_{0}$ cannot be reformulated as a power series in $z$ by interchanging the order of summations. Of course, this is a mathematical necessity: The general power function $z^{\rho}$ with $\rho \in \mathbb{R} \backslash \mathbb{N}_{0}$ is not analytic at the origin, which implies that a power series about $z=0$ cannot exist.

So far, the analysis of this Section has only produced obvious results and no new insight: The integral power $z^{m}$ with $m \in \mathbb{N}_{0}$ is analytic at the origin. Consequently, it must be possible to reformulate its finite Laguerre expansion (3.4) as a polynomial in $z$. In contrast, the nonintegral power $z^{\rho}$ with $\rho \in \mathbb{R} \backslash \mathbb{N}_{0}$ is not analytic at the origin. Accordingly, a power series for $z^{\rho}$ about $z=0$ cannot exist. At least formally, the infinite Laguerre expansion (3.3) for $z^{\rho}$ can be rearranged to yield the power series (3.7), but this power series is mathematically meaningless since almost all of its series coefficients are infinite in magnitude.

Nevertheless, the example of the general power function $z^{\rho}$ is instructive since it shows that mathematics cannot be cheated by formally rearranging Laguerre series. Therefore, we can try to use the approach described above also in the case of essentially arbitrary infinite Laguerre series of the type of (1.1). We insert the explicit expression (2.1) of the generalized Laguerre polynomial into the Laguerre series for some function, and rearrange the order of summations. As a final step, we have to analyze whether and under which conditions the inner infinite series expansions for the coefficients of the resulting the power series converge.

But first, let us consider a partial sum of the general Laguerre series (1.1):

$$
f_{N}(z)=\sum_{n=0}^{N} \lambda_{n}^{(\alpha)} L_{n}^{(\alpha)}(z), \quad N \in \mathbb{N}_{0}
$$


Such a finite sum is simply a polynomial in $z$ and it is always possible to reformulate it by interchanging the order of the nested finite summations. If we insert the explicit expression (2.1) of the generalized Laguerre polynomials into (3.10) and rearrange the order of summations, we obtain after some algebra:

$$
\begin{aligned}
f_{N}(z) & =\sum_{n=0}^{N} \lambda_{n}^{(\alpha)} \frac{(\alpha+1)_{n}}{n !} \sum_{v=0}^{n} \frac{(-n)_{v}}{(\alpha+1)_{v}} \frac{z^{v}}{v !} \\
& =\sum_{v=0}^{N} \frac{z^{v}}{(\alpha+1)_{v} v !} \sum_{n=v}^{N} \frac{(-n)_{v}(\alpha+1)_{n}}{n !} \lambda_{n}^{(\alpha)}
\end{aligned}
$$

This expression can be streamlined further, yielding

$$
f_{N}(z)=\sum_{v=0}^{N} \frac{(-z)^{v}}{v !} \sum_{\mu=0}^{N-v} \frac{(\alpha+v+1) \mu}{\mu !} \lambda_{\mu+v}^{(\alpha)} .
$$

Thus, in the case of finite Laguerre expansions of the type of (3.10), a rearrangement of the order of the nested finite summations is always possible.

Let us now consider the rearrangement of the infinite series (1.1). By inserting the explicit expression (2.1) of the generalized Laguerre polynomials into 1.1 and rearranging the order of summations, we formally obtain the following power series in $z$ :

$$
f(z)=\sum_{v=0}^{\infty} \frac{(-z)^{v}}{v !} \sum_{\mu=0}^{\infty} \frac{(\alpha+v+1) \mu}{\mu !} \lambda_{\mu+v}^{(\alpha)} .
$$

If we compare (3.13) and (3.14), we immediately see that the rearrangement of an infinite Laguerre expansion is not necessarily possible since we now have an inner infinite series instead of an inner finite sum. Accordingly, many things can go wrong if we mechanically perform the limit $N \rightarrow \infty$ in (3.13). The power series (3.14) for $f(z)$ makes sense if and only if the inner series on the right-hand side of (3.14) converges for every $v \in \mathbb{N}_{0}$. Otherwise, we have a formal power series with expansion coefficients that are infinite in magnitude. This scenario corresponds to the formal, but mathematically meaningless power series 3.7 for $z^{\rho}$ with $\rho \in \mathbb{R} \backslash \mathbb{N}_{0}$.

\section{Algebraically Decaying Series Coefficients}

In this Section, the convergence of the inner $\mu$ series in the rearranged Laguerre expansion (3.14) is analyzed by making several assumptions on the large index $(n \rightarrow \infty)$ asymptotics of the coefficients $\lambda_{n}^{(\alpha)}$.

The sufficient convergence condition $(2.18)$ shows that the coefficients $\lambda_{n}^{(\alpha)}$ in (1.1) can decay algebraically in $n$, which in practice implies (very) bad convergence. Thus, let us assume for the moment that the $\lambda_{n}^{(\alpha)}$ all have the same sign at least for sufficiently large indices $n$, and that they possess the following large index asymptotics:

$$
\lambda_{n}^{(\alpha)} \sim n^{-\beta}, \quad n \rightarrow \infty, \quad \beta>0 .
$$

For an analysis of the convergence of the inner $\mu$ series, it is helpful to rewrite (3.14) as follows:

$$
f(x)=\frac{1}{\Gamma(\alpha+1)} \sum_{v=0}^{\infty} \frac{(-x)^{v}}{(\alpha+1)_{v} v !} \sum_{\mu=0}^{\infty} \frac{\Gamma(\alpha+\mu+v+1)}{\mu !} \lambda_{\mu+v}^{(\alpha)} .
$$


We first analyze the large index asymptotics of the factor $\Gamma(\alpha+\mu+v+1) / \mu$ !. With the help of (2.16), we obtain for fixed and finite $v \in \mathbb{N}_{0}$ the following asymptotic approximation:

$$
\frac{\Gamma(\alpha+\mu+v+1)}{\mu !} \sim \mu^{\alpha+v}, \quad \mu \rightarrow \infty
$$

For fixed and finite $v \in \mathbb{N}_{0}$, the asymptotic estimate (4.1) translates to

$$
\lambda_{\mu+v}^{(\alpha)} \sim(\mu+v)^{-\beta}=\mu^{-\beta}+\mathrm{O}\left(\mu^{-\beta-1}\right), \quad \mu \rightarrow \infty .
$$

Combination of 4.3) and (4.4) yields

$$
\frac{\Gamma(\alpha+\mu+v+1)}{\mu !} \lambda_{\mu+v}^{(\alpha)} \sim \mu^{\alpha+v-\beta}, \quad \mu \rightarrow \infty .
$$

Thus, the inner $\mu$ series in (3.14) diverges at least for sufficiently large values of the outer index $v$ if the series coefficients $\lambda_{n}^{(\alpha)}$ occurring in (3.14) ultimately have the same sign and decay algebraically like a fixed power $\beta$ of the index $n$. Thus, a function represented by a Laguerre expansion with ultimately monotone and algebraically decaying series coefficients cannot be analytic in a neighborhood of the origin. This conclusion is in agreement with the remarks by Gottlieb and Orszag [49, p. 42] and by Doha [41, p. 5452], respectively, who had stated that the Laguerre series for a given function converges faster than algebraically if the function under consideration is analytic at the origin.

As discussed in more details in Section 3 , the general power function $z^{\rho}$ with $\rho \in \mathbb{R} \backslash \mathbb{N}_{0}$ is not analytic at the origin. This fact can also be deduced from its Laguerre series (3.3). With the help of 2.16), we obtain the following leading order asymptotic estimate for the coefficients in (3.3):

$$
\frac{\Gamma(-\rho+n)}{\Gamma(\alpha+n+1)} \sim n^{-\alpha-\rho-1}, \quad n \rightarrow \infty
$$

Comparison with 2.18) shows that this asymptotic estimate implies the convergence of the Laguerre series (3.3) for $z^{\rho}$ with respect to the norm (2.6) of the Hilbert space $L_{x^{\alpha}}^{2} \mathrm{e}^{-x}([0, \infty))$ if $\alpha+2 \rho>-1$ holds. However, this estimate also shows that $z^{\rho}$ with $\rho \in \mathbb{R} \backslash \mathbb{N}_{0}$ cannot be analytic at the origin $z=0$.

The convergence properties of monotone and alternating series differ substantially. A monotone series $\sum_{n=0}^{\infty} a_{n}$, whose terms all have the same sign, converges, if the series terms $a_{n}$ decay at least like $a_{n}=\mathrm{O}\left(n^{-1-\varepsilon}\right)$ with $\varepsilon>0$ as $n \rightarrow \infty$ or faster. In contrast, an alternating series $\sum_{n=0}^{\infty}(-1)^{n}\left|b_{n}\right|$ converges if the terms $b_{n}$ decrease in magnitude and approach zero as $n \rightarrow \infty$. This alone would not suffice to guarantee the convergence of the inner $\mu$ series in (3.14). However, alternating series have the undeniable advantage that summability techniques for divergent series can be employed (further details as well as numerous references can be found in Appendices $\mathrm{A}$ and $\mathrm{B}$. In this way, it is frequently possible to associate a finite value to a divergent alternating series $\sum_{n=0}^{\infty}(-1)^{n}\left|b_{n}\right|$, whose terms do not vanish as $n \rightarrow \infty$ and even grow in magnitude with increasing index.

Thus, it should be interesting to investigate whether Laguerre series of the type of 1.1 with strictly alternating series coefficients $\lambda_{n}^{(\alpha)}$, whose absolute values are at least asymptotically proportional to a fixed power of the index $n$, correspond to functions that are analytic in a neighborhood of the origin.

As an example of a Laguerre series with ultimately strictly alternating and algebraically decaying coefficients, let us consider the following Laguerre series, which differs from the 
Laguerre series $(3.3)$ for the general power function $z^{\rho}$ only by the alternating sign $(-1)^{n}$ :

$$
\begin{gathered}
G_{\rho}^{(\alpha)}(z)=\frac{\Gamma(\rho+\alpha+1)}{\Gamma(\alpha+1} \sum_{n=0}^{\infty}(-1)^{n} \frac{(-\rho)_{n}}{(\alpha+1)_{n}} L_{n}^{(\alpha)}(z), \\
\rho \in \mathbb{R} \backslash \mathbb{N}_{0}, \quad \alpha+2 \rho>-1 .
\end{gathered}
$$

The convergence condition $\alpha+2 \rho>-1$ guarantees that $G_{\rho}^{(\alpha)}(z)$ belongs just like the power function $z^{\rho}$ with $\rho \in \mathbb{R} \backslash \mathbb{N}_{0}$ to the Hilbert space $L_{x^{\alpha} \mathrm{e}^{-x}}^{2}([0, \infty))$. The case $\rho \in \mathbb{N}_{0}$ is excluded because then $G_{\rho}^{(\alpha)}$ would be a polynomial in $z$, whose analyticity at the origin is obvious.

It is, however, unclear whether $G_{\rho}^{(\alpha)}(z)$ with $\rho \in \mathbb{R} \backslash \mathbb{N}_{0}$ is analytic at the origin, i.e., whether the inner $\mu$ series in the rearranged Laguerre series (3.14) converges for arbitrary values of $v \in \mathbb{N}_{0}$. For an investigation of this question, let us define

$$
\lambda_{n}^{(\alpha)}=(-1)^{n} \frac{\Gamma(\rho+\alpha+1)}{\Gamma(-\rho)} \frac{\Gamma(-\rho+n)}{\Gamma(\alpha+n+1)} .
$$

Inserting this into the modified rearranged Laguerre expansion (4.2) yields:

$$
\begin{aligned}
G_{\rho}^{(\alpha)}(z) & =\frac{\Gamma(\rho+\alpha+1)}{\Gamma(\alpha+1)} \sum_{v=0}^{\infty} \frac{(-\rho)_{v}}{(\alpha+1)_{v}} \frac{z^{v}}{v !} \sum_{\mu=0}^{\infty}(-1)^{\mu} \frac{(-\rho+v)_{\mu}}{\mu !} \\
& =\frac{\Gamma(\rho+\alpha+1)}{\Gamma(\alpha+1)} \sum_{v=0}^{\infty} \frac{(-\rho)_{v}}{(\alpha+1)_{v}} \frac{z^{v}}{v !}{ }_{1} F_{0}(-\rho+v ;-1) .
\end{aligned}
$$

The generalized hypergeometric series ${ }_{1} F_{0}$ in 4.10 is a special case of the binomial series (3.8) which can be expressed in close form. Thus, we obtain:

$$
{ }_{1} F_{0}(-\rho+v ;-1)=\lim _{z \rightarrow-1}(1-z)^{\rho-v}=2^{\rho-v} .
$$

Since 2.16) implies $(-\rho+v)_{\mu} / \mu !=\mathrm{O}\left(\mu^{v-\rho-1}\right)$ as $\mu \rightarrow \infty$, the hypergeometric series ${ }_{1} F_{0}(-\rho+v ; z)$ converges only for $|z|<1$. Thus, ${ }_{1} F_{0}(-\rho+v ;-1)$ is - strictly speaking - undefined and a divergent series. However, $(1-z)^{n-\rho}$ remains well defined as $z \rightarrow-1$. Therefore, (4.11) essentially corresponds to an analytic continuation that implicitly uses the concepts of Abel summation which is for instance discussed in Hardy's classic book [78].

Inserting (4.11) into 4.10 yields:

$$
G_{\rho}^{(\alpha)}(z)=2^{\rho} \frac{\Gamma(\rho+\alpha+1)}{\Gamma(\alpha+1)}{ }_{1} F_{1}(-\rho ; \alpha+1 ; z / 2) .
$$

A confluent hypergeometric series ${ }_{1} F_{1}(a ; b ; z)$ converges absolutely for all complex $a$, $b$, and $z$ as long as $-b \notin \mathbb{N}_{0}$ (see for example [119, p. 2]). Thus, for $-b \notin \mathbb{N}_{0}$ such a ${ }_{1} F_{1}$ is an entire function in $z$. Since we always assume $\alpha>-1, G_{\rho}^{(\alpha)}(z)$ is in every neighborhood of the origin $z=0$ an analytic function. Thus, (4.12) shows that the remarks by Gottlieb and Orszag [49. p. 42] and by Doha [41, p. 5452], who stated that such a Laguerre series converges faster than algebraically if the function under consideration is analytic at the origin, is imprecise.

If the argument $z$ of a confluent hypergeometric function ${ }_{1} F_{1}(a ; b, z)$ is real and approaches $+\infty$, then we have the following asymptotic behavior (see for example [119, Eq. (4.1.7)]):

$$
{ }_{1} F_{1}(a ; b ; z)=\frac{\Gamma(a)}{\Gamma(b)} \mathrm{e}^{z} z^{a-b}[1+\mathrm{O}(1 / z)], \quad z \rightarrow+\infty .
$$


This asymptotic estimate shows that the integral

$$
\begin{aligned}
\int_{0}^{\infty} \mathrm{e}^{-z} z^{\alpha}\left[G_{\rho}^{(\alpha)}(z)\right]^{2} \mathrm{~d} z & \\
& =\left[2^{\rho} \frac{\Gamma(\rho+\alpha+1)}{\Gamma(\alpha+1)}\right]^{2} \int_{0}^{\infty} \mathrm{e}^{-z} z^{\alpha}\left[{ }_{1} F_{0}(-\rho ; \alpha+1 ; z / 2)\right]^{2} \mathrm{~d} z
\end{aligned}
$$

converges if $\alpha>-1$ and $\alpha+2 \rho>-1$ hold. At the lower integration limit $z=0$, the integrand behaves like $z^{\alpha}$, which requires $\alpha>-1$, and at the upper integration limit $z=\infty$, the integrand behaves like $z^{-\alpha-2 \rho-2}$, which requires $\alpha+2 \rho>-1$. Thus, $G_{\rho}^{(\alpha)}(z)$ belongs for $\alpha>-1$ and $\alpha+2 \rho>-1$ to the Hilbert space $L_{z^{\alpha} \mathrm{e}^{-z}}^{2}([0, \infty))$ defined by $[2.7)$.

It is possible to check the correctness of the power series representation 4.12) for $G_{\rho}^{(\alpha)}(z)$ by expanding it in terms of generalized Laguerre polynomials. According to $1.1 \mathrm{~b}$, we then have to compute the following inner product:

$$
\begin{aligned}
\lambda_{n}^{(\alpha)}= & 2^{\rho} \frac{n !}{\Gamma(\alpha+n+1)} \frac{\Gamma(\rho+\alpha+1)}{\Gamma(\alpha+1)} \\
& \times \int_{0}^{\infty} \mathrm{e}^{-z} z^{\alpha} L_{n}^{(\alpha)}(z){ }_{1} F_{1}(-\rho ; \alpha+1 ; z / 2) \mathrm{d} z
\end{aligned}
$$

Since the confluent hypergeometric function ${ }_{1} F_{1}$ in 4.15 is analytic in every neighborhood of the origin, integration and summation can be interchanged. We now use [50, Eq. (7.414.11) on p. 850]

$$
\int_{0}^{\infty} \mathrm{e}^{-t} t^{\gamma-1} L_{n}^{(\mu)}(t) \mathrm{d} t=\frac{\Gamma(\gamma) \Gamma(1+\mu-\gamma+n)}{n ! \Gamma(1+\mu-\gamma)}, \quad \operatorname{Re}(\gamma)>0
$$

to obtain:

$$
\begin{gathered}
\int_{0}^{\infty} e^{-z} z^{\alpha} L_{n}^{(\alpha)}(z){ }_{1} F_{1}(-\rho ; \alpha+1 ; z / 2) \mathrm{d} z \\
=\frac{\Gamma(\alpha+1)}{n !} \sum_{m=0}^{\infty} \frac{(-\rho)_{m}(-m)_{n}}{m !} 2^{-m}
\end{gathered}
$$

The fact that the Pochhammer symbol $(-m)_{n}$ with $m, n \in \mathbb{N}_{0}$ satisfies $(-m)_{n}=0$ for $m=$ $0,1, \ldots, n-1$ suggest the substitution $m \rightarrow n+v$, yielding:

$$
\begin{aligned}
& \int_{0}^{\infty} e^{-z} z^{\alpha} L_{n}^{(\alpha)}(z){ }_{1} F_{1}(-\rho ; \alpha+1 ; z / 2) \mathrm{d} z \\
& \quad=(-1)^{n} \frac{\Gamma(\alpha+1)(-\rho)_{n}}{2^{n} n !}{ }_{1} F_{0}(-\rho+n ; 1 / 2) .
\end{aligned}
$$

With the help of (3.8) we obtain

$$
{ }_{1} F_{0}(-\rho+n ; 1 / 2)=(1 / 2)^{\rho-n}=2^{n-\rho} .
$$

Combination of (4.18) and 4.19) then yields:

$$
\begin{gathered}
\int_{0}^{\infty} e^{-z} z^{\alpha} L_{n}^{(\alpha)}(z){ }_{1} F_{1}(-\rho ; \alpha+1 ; z / 2) \mathrm{d} z \\
=\frac{(-1)^{n}}{2^{\rho}} \frac{\Gamma(\alpha+1)(-\rho)_{n}}{n !}
\end{gathered}
$$


We also obtain this result if we combine 4.8) and 4.15).

As a more complicated example, let us now consider the following Laguerre series:

$$
2^{\mathscr{H}_{1}^{(\alpha)}}(a, b ; c ; z)=\sum_{n=0}^{\infty}(-1)^{n} \frac{(a)_{n}(b)_{n}}{(c)_{n}(\alpha+1)_{n}} L_{n}^{(\alpha)}(z) .
$$

Concerning the real parameters $a, b$, and $c$, we assume for the moment only that this series exists, which requires $-c \notin \mathbb{N}_{0}$, that it does not terminate, which requires $-a,-b \notin \mathbb{N}_{0}$. and that it converges with respect to the norm (2.6) of the Hilbert space $L_{z^{\alpha} \mathrm{e}^{-z}}^{2}([0, \infty))$. It follows from (2.16) that this is the case if $c-a-b+(\alpha+1) / 2>0$.

For an investigation of the analyticity of ${ }_{2} \mathscr{H}_{1}^{(\alpha)}(a, b ; c ; z)$ at the origin, let us define

$$
\lambda_{n}^{(\alpha)}=(-1)^{n} \frac{(a)_{n}(b)_{n}}{(c)_{n}(\alpha+1)_{n}} .
$$

By proceeding as in the case of the Laguerre series 4.7) for $G_{\rho}^{(\alpha)}(z)$ we obtain after a short calculation:

$$
{ }_{2} \mathscr{H}_{1}^{(\alpha)}(a, b ; c ; z)=\sum_{v=0}^{\infty}{ }_{2} F_{1}(a+v, b+v ; c+v ;-1) \frac{(a)_{v}(b)_{v}}{(c)_{v} v !} \frac{z^{v}}{(\alpha+1)_{v}} .
$$

It follows from 2.16) that $\Gamma(a+v+n) \Gamma(b+v+n) /[\Gamma(c+v+n) n !] \sim n^{a+b+v-c-1}$ as $n \rightarrow \infty$. Consequently, the Gaussian hypergeometric series ${ }_{2} F_{1}(a+v, b+v ; c+v ; z)$ with $z=-1$ diverges for all sufficiently large $v \in \mathbb{N}_{0}$. Nevertheless, it is possible to associate a finite value to the hypergeometric function corresponding to this divergent series with the help of the following two linear transformations [102, p. 47]:

$$
\begin{aligned}
{ }_{2} F_{1}(a, b ; c ; z) & =(1-z)^{-a}{ }_{2} F_{1}(a, c-b ; c ; z /(z-1)) \\
& =(1-z)^{-b}{ }_{2} F_{1}(c-a, b ; c ; z /(z-1)) .
\end{aligned}
$$

The transformation $z \rightarrow z^{\prime}=z /(z-1)$ maps $z=-1$, which is located on the boundary of the circle of convergence, to $z^{\prime}=1 / 2$, which is located in the interior of the circle of convergence. Thus, the in general divergent hypergeometric series in (4.23) can be replaced by a convergent hypergeometric series according to

$$
\begin{aligned}
{ }_{2} F_{1}(a+v, b+v ; c+v ;-1) & =2^{-a-v}{ }_{2} F_{1}(a+v, c-b ; c+v ; 1 / 2) \\
& =2^{-b-v}{ }_{2} F_{1}(c-a, b+v ; c+v ; 1 / 2) .
\end{aligned}
$$

Inserting (4.26) and (4.27) into (4.23) yields two equivalent power series expansions for the function defined by the Laguerre series 4.21 , which seem to be new:

$$
\begin{aligned}
2^{\mathscr{H}_{1}}{ }^{(\alpha)}(a, b ; c ; z) & =2^{-a} \sum_{v=0}^{\infty}{ }_{2} F_{1}(a+v, c-b ; c+v ; 1 / 2) \frac{(a)_{v}(b)_{v}}{(c)_{v} v !} \frac{(z / 2)^{v}}{(\alpha+1)_{v}} \\
& =2^{-b} \sum_{v=0}^{\infty}{ }_{2} F_{1}(c-a, b+v ; c+v ; 1 / 2) \frac{(a)_{v}(b)_{v}}{(c)_{v} v !} \frac{(z / 2)^{v}}{(\alpha+1)_{v}}
\end{aligned}
$$

A detailed analysis of the domain of analyticity of ${ }_{2} \mathscr{H}_{1}^{(\alpha)}(a, b ; c ; z)$ requires asymptotic estimates of the behavior of the Gaussian hypergeometric series in (4.23), 4.28) and (4.29) as $v \rightarrow \infty$. If we use the linear transformation [102, p. 47]

$$
{ }_{2} F_{1}(a, b ; c ; z)=(1-z)^{c-a-b}{ }_{2} F_{1}(c-a, c-b ; c ; z),
$$


which certainly holds for $|z|<1$, we obtain:

$$
{ }_{2} F_{1}(a+v, b+v ; c+v ; z)=(1-z)^{c-a-b-v}{ }_{2} F_{1}(c-a, c-b ; c+v ; z) .
$$

The asymptotic estimate (2.16) yields $\Gamma(c-a+n) \Gamma(c-b+n) /[\Gamma(c+v+n) n !] \sim n^{c-a-b-v-1}$ as $n \rightarrow \infty$. Accordingly, the hypergeometric series on the right-hand side of (4.31) converges at least for sufficiently large values of $v$ also for $z=-1$ and provides an analytic continuation. Moreover, (4.31) is a convenient starting point for the construction of an asymptotic expansion of the divergent hypergeometric series in (4.23) as $v \rightarrow \infty$ (compare also [127, Eq. (15)]):

$$
\begin{gathered}
{ }_{2} F_{1}(a+v, b+v ; c+v ;-1)=2^{c-a-b-v} \sum_{n=0}^{\infty}(-1)^{n} \frac{(c-a)_{n}(c-b)_{n}}{(c+v)_{n} n !} \\
=2^{c-a-b-v}\left[1-\frac{(c-a)(c-b)}{(c+v)}+\mathrm{O}\left(v^{-2}\right)\right], \quad v \rightarrow \infty .
\end{gathered}
$$

This asymptotic estimate shows that ${ }_{2} \mathscr{H}_{1}^{(\alpha)}(a, b ; c ; z)$ is for $-c \notin \mathbb{N}_{0}$ analytic in every neighborhood of the origin.

\section{Exponentially and Factorially Decaying Series Coefficients}

It is immediately obvious that the inner $\mu$ series in (3.14) converges if the series coefficients $\lambda_{n}^{(\alpha)}$ decay for sufficiently large indices $n$ exponentially, satisfying for instance

$$
\lambda_{n}^{(\alpha)} \sim n^{\theta} R^{n}, \quad \theta \in \mathbb{R}, \quad|R|<1, \quad n \rightarrow \infty .
$$

As the probably most simple example of a Laguerre series of the type of 1.1) with exponentially decaying coefficients, let us consider the following expansion:

$$
\mathscr{E}^{(\alpha)}(t ; z)=\sum_{n=0}^{\infty} t^{n} L_{n}^{(\alpha)}(z) .
$$

It is immediately obvious that the Laguerre series (5.2) converges in the mean with respect to the norm (2.6) of the Hilbert space $L_{z^{\alpha} \mathrm{e}^{-z}}^{2}([0, \infty))$ for $|t|<1$, and that it diverges for $|t| \geq 1$. Equivalently, we can say that the series (2.13) for the norm $\|\mathscr{E}(\alpha)(t ; z)\|_{z^{\alpha} \mathrm{e}^{-z, 2}}$ produces a finite result for $|t|<1$ and that it diverges for $|t| \geq 1$.

Ignoring for the moment all questions of convergence, let us now assume that $t$ is an unspecified complex number and insert $\lambda_{n}^{(\alpha)}=t^{n}$ into (3.14). Then, we obtain:

$$
\begin{aligned}
\mathscr{E}^{(\alpha)}(t ; z) & =\sum_{v=0}^{\infty} \frac{(-t z)^{v}}{v !} \sum_{\mu=0}^{\infty} \frac{(\alpha+v+1) \mu}{\mu !} t^{\mu} \\
& =\sum_{v=0}^{\infty} \frac{(-t z)^{v}}{v !}{ }_{1} F_{0}(\alpha+v+1 ; t) .
\end{aligned}
$$

A nonterminating hypergeometric series ${ }_{1} F_{0}(a ; z)$ converges only in the interior of the unit circle. Since we always assume $\alpha>-1$, the hypergeometric series ${ }_{1} F_{0}(\alpha+v+1 ; t)$ in (5.4) does not terminate and we have to require $|t|<1$. However, ${ }_{1} F_{0}(\alpha+v+1 ; t)$ can according to 3.8 be expressed in closed form,

$$
{ }_{1} F_{0}(\alpha+v+1 ; t)=(1-t)^{-\alpha-v-1}, \quad t \in \mathbb{C} \backslash\{1\},
$$


which yields an analytic continuation for $t \neq 1$. Inserting (5.5) into (5.4) yields:

$$
\begin{aligned}
\mathscr{E}^{(\alpha)}(t ; z) & =(1-t)^{-\alpha-1} \sum_{v=0}^{\infty} \frac{(-t z /[1-t])^{v}}{v !} \\
& =(1-t)^{-\alpha-1} \exp (-t z /[1-t]) .
\end{aligned}
$$

Accordingly, the Laguerre series (5.2) for $\mathscr{E}^{(\alpha)}(t ; z)$ is nothing but the well known generating function [102, p. 242]

$$
\sum_{n=0}^{\infty} L_{n}^{(\alpha)}(z) t^{n}=(1-t)^{-\alpha-1} \exp (z t /[t-1]), \quad|t|<1,
$$

in disguise.

As a generalization of the alternating Laguerre series (4.7), let us consider the following expansion with exponentially decaying coefficients:

$$
\begin{gathered}
\mathscr{G}_{\rho}^{(\alpha)}(s ; z)=\frac{\Gamma(\rho+\alpha+1)}{\Gamma(\alpha+1)} \sum_{n=0}^{\infty}(-s)^{n} \frac{(-\rho)_{n}}{(\alpha+1)_{n}} L_{n}^{(\alpha)}(z), \\
\rho \in \mathbb{R} \backslash \mathbb{N}_{0}, \quad \alpha+2 \rho>-1 .
\end{gathered}
$$

Obviously, this Laguerre series converges in the mean for $|s|<1$.

For an investigation of the analyticity of $\mathscr{G}_{\rho}^{(\alpha)}(s ; z)$ at the origin, let us define

$$
\lambda_{n}^{(\alpha)}=\frac{\Gamma(\rho+\alpha+1)}{\Gamma(-\rho)} \frac{\Gamma(-\rho+n)}{\Gamma(\alpha+n+1)}(-s)^{n} .
$$

Inserting this into the modified rearranged Laguerre expansion (4.2) yields:

$$
\begin{aligned}
\mathscr{G}_{\rho}^{(\alpha)}(s ; z) & =\frac{\Gamma(\rho+\alpha+1)}{\Gamma(\alpha+1)} \sum_{v=0}^{\infty} \frac{(-\rho)_{v}}{(\alpha+1)_{v}} \frac{(s z)^{v}}{v !} \sum_{\mu=0}^{\infty} \frac{(-\rho+v)_{\mu}}{\mu !}(-s)^{\mu} \\
& =\frac{\Gamma(\rho+\alpha+1)}{\Gamma(\alpha+1)} \sum_{\nu=0}^{\infty} \frac{(-\rho)_{v}}{(\alpha+1)_{v}} \frac{(s z)^{v}}{v !}{ }_{1} F_{0}(-\rho+v ;-s) .
\end{aligned}
$$

With the help of (3.8), we obtain

$$
{ }_{1} F_{0}(-\rho+v ;-s)=(1+s)^{\rho-v}, \quad s \neq-1 .
$$

Inserting (5.13) into 5.12) yields:

$$
\mathscr{G}_{\rho}^{(\alpha)}(s ; z)=(1+s)^{\rho} \frac{\Gamma(\rho+\alpha+1)}{\Gamma(\alpha+1)}{ }_{1} F_{1}(-\rho ; \alpha+1 ; s z /[1+s]) .
$$

The argument of the confluent hypergeometric series ${ }_{1} F_{1}$ becomes infinite for $s=-1$. If we assume $\alpha>-1$, then the right-hand side of (5.14) is mathematically meaningful for all $z \in \mathbb{C}$ and for all $s \in \mathbb{C} \backslash\{-1\}$. However, this is not true for the corresponding Laguerre series (5.9). The convergence of the series $(2.13)$ for the norm $\left\|\mathscr{G}_{\rho}^{(\alpha)}(s ; z)\right\|_{z^{\alpha} \mathrm{e}^{-z, 2}}$ is only guaranteed for $|s|<1$.

It follows from (5.14) that $\mathscr{G}_{\rho}^{(\alpha)}(s ; z)$ is essentially a confluent hypergeometric series ${ }_{1} F_{1}$. Accordingly, the Laguerre series (5.9) for $\mathscr{G}_{\rho}^{(\alpha)}(s ; z)$ can be rewritten as follows:

$$
{ }_{1} F_{1}(-\rho ; \alpha+1 ; s z /[1+s])=(1+s)^{-\rho} \sum_{n=0}^{\infty}(-s)^{n} \frac{(-\rho)_{n}}{(\alpha+1)_{n}} L_{n}^{(\alpha)}(z)
$$


If we make in (5.15) the substitutions $\rho \rightarrow-c$ and $s \rightarrow-t$, we see that the function $\mathscr{G}_{\rho}^{(\alpha)}(s ; z)$ is nothing but the well known generating function [109, Eq. (3) on p. 202])

$$
\sum_{n=0}^{\infty} \frac{(c)_{n} L_{n}^{(\alpha)}(x)}{(\alpha+1)_{n}} t^{n}=(1-t)^{-c}{ }_{1} F_{1}(c ; \alpha+1 ;-x t /[1-t])
$$

in disguise.

If we set $s=-1$ in the Laguerre series $(5.9)$ for $\mathscr{G}_{\rho}^{(\alpha)}(s ; z)$, we obtain the Laguerre series (3.3) for $z^{\rho}$. To analyze the behavior of $\mathscr{G}_{\rho}^{(\alpha)}(s ; z)$ as $s \downarrow-1$, we write $s=-1+\delta$ with $\delta \geq 0$ and consider in (5.14) the limit $\delta \downarrow 0$. We obtain

$$
\mathscr{G}_{\rho}^{(\alpha)}(\delta-1 ; z)=\delta^{\rho} \frac{\Gamma(\rho+\alpha+1)}{\Gamma(\alpha+1)}{ }_{1} F_{1}(-\rho ; \alpha+1 ;(\delta-1) z / \delta) .
$$

Next, we use the following asymptotic estimate [119, Eq. (4.1.8)]:

$$
{ }_{1} F_{1}(a ; b ; z)=\frac{\Gamma(b)}{\Gamma(b-a)}(-z)^{-a}[1+\mathrm{O}(1 / z)], \quad z \rightarrow-\infty .
$$

If we insert 5.18) into 5.17, we obtain in the limit of vanishing $\delta \geq 0$ :

$$
\begin{aligned}
\mathscr{G}_{\rho}^{(\alpha)}(-1 ; z) & =\lim _{\delta \downarrow 0} \mathscr{G}_{\rho}^{(\alpha)}(\delta-1 ; z) \\
& =\lim _{\delta \downarrow 0}[(1-\delta) z]^{\rho}\left\{1+\mathrm{O}\left(\frac{\delta}{(\delta-1) z}\right)\right\}=z^{\rho} .
\end{aligned}
$$

Thus, $\mathscr{G}_{\rho}^{(\alpha)}(s ; z)$ possesses the one-sided limit $z^{\rho}$ as $s \downarrow-1$, as it should according to the Laguerre series (3.3) and (5.9).

The transformation formula (3.14) can only be used if the function represented by the Laguerre series is analytic at the origin, because otherwise the inner $\mu$ series diverges. Thus, it is not possible to obtain in this way an explicit expression for a nonanalytic function defined by a Laguerre series of the type of (1.1) with monotone and algebraically decaying series coefficients $\lambda_{n}^{(\alpha)}$.

However, the fact that the in general nonanalytic power function $z^{\rho}$ can according to 5.19) be obtained by considering the one-sided limit $s \downarrow-1$ in the analytic function $\mathscr{G}_{\rho}^{(\alpha)}(s ; z)$ indicates that the situation is not as hopeless as it may look at first sight.

Let us therefore assume for the moment that the coefficients $\lambda_{n}^{(\alpha)}$ of a Laguerre series are monotone and decay algebraically as $n \rightarrow \infty$. This implies that the inner $\mu$ series in (3.14) produce infinities. However, we could try to apply the transformation formula (3.14) to a modified Laguerre series with coefficients $t^{n} \lambda_{n}^{(\alpha)}$. Since these coefficients decay exponentially for $|t|<1$, this modified Laguerre series represents an analytic function. If we succeed in constructing an explicit expression for this analytic function, then we can try to perform the one-sided limit $t \uparrow 1$ in this expression. If this can be done, we obtain an explicit expression for a nonanalytic function. Obviously, this idea deserves to be investigated further.

Although the explicit expression (5.14) for $\mathscr{G}_{\rho}^{(\alpha)}(s ; z)$ is mathematically well defined for all $s \in \mathbb{C} \backslash\{-1\}$ and possesses a one-sided limit for $s \downarrow-1$, its Laguerre series (5.9) requires more restrictive conditions. In general, generating functions of the kind of 5.9) converge only for $|s|<1$, i.e., in the interior of the unit circle, and possibly also for some points on the boundary of the unit circle. 
If we make in (5.9) or in (5.15) the substitution $s \rightarrow 1$, we obtain the series expansions (4.7) and 4.12), respectively, for $G_{\rho}^{(\alpha)}(x)$. Thus, in this case it is possible to extend the Laguerre series (5.9), which converges exponentially in the mean for $|s|<1$, to the boundary of its circle of convergence, yielding a convergent Laguerre series with algebraically decaying coefficients that ultimately have strictly alternating signs.

It is important to note that this approach does not always work. If we make in the Laguerre series (5.2), which because of (5.7) is nothing but the generating function (5.8), the substitution $t \rightarrow-1$, we formally obtain:

$$
\mathrm{e}^{z / 2}=2^{\alpha+1} \sum_{n=0}^{\infty}(-1)^{n} L_{n}^{(\alpha)}(z) .
$$

However, $\mathrm{e}^{z / 2}$ does not belong to the Hilbert space $L_{z^{\alpha} \mathrm{e}^{-z}}^{2}([0, \infty))$ defined by (2.7), and it follows from (2.18) that the series (2.12) for the norm $\left\|\mathrm{e}^{z / 2}\right\|_{z^{\alpha} \mathrm{e}^{-z, 2}}$ does not converge.

It is also not possible to assume $|s|>1$ in the Laguerre series (5.9) for $\mathscr{G}_{\rho}^{(\alpha)}(s ; z)$, because then the series $(2.12)$ for the norm $\left\|\mathscr{G}_{\rho}^{(\alpha)}(s ; z)\right\|_{z^{\alpha} \mathrm{e}^{-z, 2}}$ diverges. In contrast, the explicit expression (5.14) for $\mathscr{G}_{\rho}^{(\alpha)}(s ; z)$ remains meaningful for $|s|>1$.

If the series coefficients of a power series decay factorially, we can expect that the function represented by this power series is analytic in the whole complex plane $\mathbb{C}$. The most obvious examples are the exponential $\exp (z)$, or - slightly more general - the confluent hypergeometric series ${ }_{1} F_{1}(a ; b ; z)$. In contrast, power series, whose coefficients decay algebraically, converge only in compact subset of the complex plane. It should be of interest to study how factorially decaying series coefficients $\lambda_{n}^{(\alpha)}$ influence the analyticity properties of functions represented by a Laguerre series of the type of (1.1).

As an example, let us consider the following Laguerre series:

$$
\mathscr{F}^{(\alpha)}(s ; z)=\sum_{n=0}^{\infty} \frac{s^{n}}{(\alpha+1)_{n}} L_{n}^{(\alpha)}(z) \text {. }
$$

Thus, we choose $\lambda_{n}^{(\alpha)}=s^{n} /(\alpha+1)_{n}$ in 11.1). Inserting this into the modified rearranged Laguerre expansion (4.2) yields:

$$
\begin{aligned}
\mathscr{F}^{(\alpha)}(s ; z) & =\sum_{v=0}^{\infty} \frac{(-s z)^{v}}{(\alpha+1)_{v} v !} \sum_{\mu=0}^{\infty} \frac{s^{\mu}}{\mu !} \\
& =\mathrm{e}^{s} \sum_{v=0}^{\infty} \frac{(-s z)^{v}}{(\alpha+1)_{v} v !}
\end{aligned}
$$

The infinite series in (5.22) can be expressed as a generalized hypergeometric series ${ }_{0} F_{1}$. We then obtain:

$$
\mathscr{F}^{(\alpha)}(s ; z)=\mathrm{e}_{0}^{s} F_{1}(\alpha+1 ;-s z) .
$$

However, this is nothing but a known generating function [102, p. 242].

The generalized hypergeometric series ${ }_{0} F_{1}(\alpha+1 ;-s z)$ in (5.24) obviously converges for all $s, z \in \mathbb{C}$ as long as $\alpha+1$ is not a negative integer. Since we always assume $\alpha>-1$, we thus can conclude that $\mathscr{F}^{(\alpha)}(s ; z)$ is an analytic function in the sense of complex analysis in both $s$ and $z$. Moreover, the series (2.13) for the norm $\left\|\mathscr{F}^{(\alpha)}(s ; z)\right\|_{z^{\alpha} \mathrm{e}^{-z, 2}}$ converges for arbitrary $s \in \mathbb{C}$. 


\section{Computational Approaches}

The examples considered in Sections 4 and 5 show that it is indeed possible to transform a Laguerre series of the type of (1.1) with the help of (3.14 to a power series about $z=0$ of the type of (1.3). The essential requirement is that the inner $\mu$ series in (3.14) has to converge. This is the case if the coefficients $\lambda_{n}^{(\alpha)}$ of the Laguerre series decay sufficiently rapidly, or - if the coefficients $\lambda_{n}^{(\alpha)}$ only decay algebraically in magnitude - ultimately have strictly alternating signs.

In Section 5 the transformation of Laguerre series with exponentially and factorially decaying series coefficients was discussed. Apart from finite Laguerre expansions, this is pretty much the best scenario which can occur in this context. The convergence of the inner $\mu$ series is guaranteed, and often such a $\mu$ series converges rapidly, making the resulting power series expansion computationally useful even if it is not possible to find a convenient closed form expression for the inner $\mu$ series.

As discussed in Section 4 , the most challenging and therefore also most interesting problems occur if the coefficients $\lambda_{n}^{(\alpha)}$ decay algebraically as $n \rightarrow \infty$. If algebraically decaying coefficients $\lambda_{n}^{(\alpha)}$ ultimately have the same sign, the inner $\mu$ series in (3.14) diverges, and it is also not possible to sum these series to something finite. Accordingly, the transformation formula (3.14) leads to a power series expansion having infinitely many series coefficients that are infinite in magnitude. This simply means that a power series in $z$ does not exist because the function under consideration is not analytic at the origin.

The inner $\mu$ series in (3.14) also does not converge if the coefficients $\lambda_{n}^{(\alpha)}$ decay algebraically and ultimately have strictly alternating signs. But in this case, suitable summation techniques are capable of associating something finite to the divergent inner $\mu$ series.

In all examples considered in Section 4 it was possible to sum the divergent inner $\mu$ series with ultimately strictly alternating signs by means of explicit analytic continuation formulas for hypergeometric series ${ }_{1} F_{0}$ and ${ }_{2} F_{1}$, respectively. For example, the Laguerre series (4.7) for $G_{\rho}^{(\alpha)}(z)$ leads according to (4.10) to an inner $\mu$ series that can be expressed as a binomial series ${ }_{1} F_{0}(-\rho+v ;-1)$ that diverges for fixed $\rho \in \mathbb{R} \backslash \mathbb{N}_{0}$ at least for sufficiently large values of $v \in \mathbb{N}_{0}$. Nevertheless, the summation of this divergent series is trivial because every hypergeometric series ${ }_{1} F_{0}(a ; z)$ with $z \neq 1$ possesses according to (3.8) a very simple closed form expression which accomplishes the necessary analytic continuation.

Similarly, the Laguerre series 4.21) for ${ }_{2} \mathscr{H}_{1}^{(\alpha)}(a, b ; c ; z)$ leads according to (4.23) to an inner $\mu$ series that can be expressed as a hypergeometric series ${ }_{2} F_{1}(a+v, b+v ; c+v ;-1)$ that also diverges for sufficiently large $v \in \mathbb{N}_{0}$. But again, it is almost trivially simple to find explicit analytic continuation formulas that replace this divergent series by convergent expansions. The linear transformations (4.24) and (4.25) do the job.

Unfortunately, the situation is not nearly as nice if the series coefficients $\lambda_{n}^{(\alpha)}$ have a more complicated structure. Let us for example consider the following Laguerre series with coefficients $\lambda_{n}^{(\alpha)}$ that are ratios of $p+1$ numerator and $p+1$ denominator Pochhammer symbols:

$$
\begin{aligned}
{ }_{p+1} \mathscr{H}_{p}^{(\alpha)}\left(a_{1}, \ldots, a_{p+1} ; b_{1}, \ldots, b_{p} ; z\right) & \\
= & \sum_{n=0}^{\infty}(-1)^{n} \frac{\left(a_{1}\right)_{n} \ldots\left(a_{p+1}\right)_{n}}{\left(b_{1}\right)_{n} \ldots\left(b_{p}\right)_{n}} \frac{L_{n}^{(\alpha)}(z)}{(\alpha+1)_{n}}, \quad p \in \mathbb{N}_{0} .
\end{aligned}
$$

Obviously, this Laguerre series generalizes the Laguerre series 4.21) for ${ }_{2} \mathscr{H}_{1}^{(\alpha)}(a, b ; c ; z)$. 
It is easy to show that the application of the transformation formula (3.14) to the Laguerre series (6.1) leads to inner $\mu$ series that can be expressed as generalized hypergeometric series

$$
{ }_{p+1} F_{p}\left(\begin{array}{c}
a_{1}+v, \ldots, a_{p+1}+v \\
b_{1}+v, \ldots, b_{p}+v
\end{array} ; z\right)=\sum_{n=0}^{\infty} \frac{\left(a_{1}+v\right)_{n} \cdots\left(a_{p+1}+v\right)_{n}}{\left(b_{1}+v\right)_{n} \cdots\left(b_{p}+v\right)_{n}} \frac{z^{n}}{n !}
$$

with argument $z=-1$. Just like the Gaussian hypergeometric series ${ }_{2} F_{1}$, a nonterminating generalized hypergeometric series ${ }_{p+1} F_{p}$ converges only in the interior of the unit circle. Moreover, 2.16 implies

$$
\frac{\Gamma\left(a_{1}+v+n\right) \Gamma\left(a_{p+1}+v+n\right)}{\Gamma\left(b_{1}+v+n\right) \Gamma\left(b_{p}+v+n\right) n !} \sim n^{a_{1}+\cdots+a_{p+1}+v-b_{1}+\cdots-b_{p}}, \quad n \rightarrow \infty .
$$

This asymptotic estimate shows that the generalized hypergeometric series (6.2) with argument $z=-1$ diverge for sufficiently large values of $v$ and have to be summed. Unfortunately, the theory of the generalized hypergeometric series ${ }_{p+1} F_{p}$ with $p \geq 2$ is not nearly as highly developed as the theory of the Gaussian hypergeometric series ${ }_{2} F_{1}$. Many transformation and/or analytic continuation formulas for a generalized hypergeometric series ${ }_{p+1} F_{p}$ with $p \geq 2$ are either not known or at least much more complicated than the corresponding expressions for a Gaussian hypergeometric series ${ }_{2} F_{1}$ (compare also [25]). We can safely assume that our chances of finding convenient explicit analytic continuation formulas, that can accomplish the summation of the divergent generalized hypergeometric series (6.2), are rapidly approaching zero with increasing $p$.

Thus, the rearrangement of Laguerre series via (3.14) can only produce closed form expressions if algebraically decaying and ultimately strictly alternating series coefficients $\lambda_{n}^{(\alpha)}$ have an exceptionally simple structure, as for example the ones in the Laguerre series (4.7) and 4.21). In the case of more complicated coefficients $\lambda_{n}^{(\alpha)}$, the necessary summations of the divergent inner $\mu$ series will be too difficult to produce closed form expressions of manageable complexity.

These and related considerations may tempt a skeptical reader to argue with some justification that all examples considered in Section 4 are actually fairly simple. Therefore, it is by no means obvious whether the approach of this article is capable of producing anything new beyond a rederivation of known and comparatively simple formulas.

While the first conclusion is certainly correct, the second is in my opinion overly pessimistic. The reason is that summation via analytic continuation formulas is not the only possibility: We can also try to use numerical summation techniques. Of course, knowing only numerical approximations to the leading coefficients of a power series is not nearly as nice as knowing explicit and possibly even simple expressions for the series coefficients, but it is certainly better than nothing. In practical applications, this limited information may suffice.

The use of numerical summation techniques is by no means a new idea. Already in 1882 , Hölder [80] proposed - inspired by an article by Frobenius [47] - to determine the value of a power series on the boundary of its circle of convergence with the help of numerical summation processes based on weighted arithmetic means.

As discussed in Appendix B] the numerical processes proposed by Hölder [80] and others subsequently evolved to a sophisticated mathematical theory of so-called regular matrix transformations that have many advantageous theoretical features and that can be used for the summation of divergent alternating series possessing similar divergence properties as the generalized hypergeometric series 6.2.

Unfortunately, regular matrix transformations are in general at best moderately powerful. Therefore, I prefer to use instead nonlinear sequence transformations, whose theoretical prop- 
erties are not yet completely understood, but which are according to experience often much better suited to achieve highly accurate summation results.

It may be interesting to note that summation techniques such as conformal mappings, reexpansions, and Padé approximants were discussed in an article by Skorokhodov [116] on the analytic continuation of a divergent generalized hypergeometric series ${ }_{p+1} F_{p}$ with argument $|z| \geq 1$. Skorokhodov completely ignored the theoretically much simpler, but also less powerful regular matrix transformations. Nevertheless, I do not think that the summation techniques considered by Skorokhodov give best results in the case of the divergent, but summable inner $\mu$ series occurring in this article. In my opinion, (much) better results can be obtained with the help of those nonlinear sequence transformations that are reviewed in Appendix B.

In this article, I apply as numerical summation techniques Wynn's epsilon algorithm (B.4), and the two Levin-type transformations (B.14) and (B.15). Wynn's epsilon algorithm produces Padé approximants according to (B.5) if the input data are the partial sums $f_{n}(z)=\sum_{k=0}^{n} \gamma_{k} z^{k}$ of a power series. The two Levin-type transformations (B.14) and B.15) are based on the remainder estimate (B.13) which corresponds to the first term neglected in the partial sum. Both are known to be highly effective in the case of both convergent and divergent alternating series.

The discussion in Section 4 should provide convincing evidence that for $z=-1$ and in particular for large values of $v \in \mathbb{N}_{0}$ it is not a particularly good idea to use the hypergeometric series ${ }_{2} F_{1}(a+v, b+v ; c+v ; z)$ for the evaluation of the hypergeometric function it represents. With the help of analytic continuation formulas like (4.24) and (4.25) or also (4.30), computationally much more convenient hypergeometric series can be derived. However, these simplifications are only possible in the case of a Gaussian hypergeometric series ${ }_{2} F_{1}$, but not necessarily in the case of more complicated generalized hypergeometric series ${ }_{p+1} F_{p}$, let alone in the case of divergent, but summable inner $\mu$ series that result from purely numerical Laguerre series coefficients $\lambda_{n}^{(\alpha)}$. Thus, the hypergeometric series ${ }_{2} F_{1}(a+v, b+v ; c+v ;-1)$ serves as a model problem for other divergent alternating series whose terms also increase in magnitude like a fixed power of the index. It should be interesting to see how much can be accomplished by employing powerful nonlinear sequence transformations.

In Tables 1 and 2, the nonlinear transformations mentioned above are applied to the partial sums

$$
s_{n}=s_{n}(a, b, c, v)=\sum_{k=0}^{n}(-1)^{k} \frac{(a+v)_{k}(b+v)_{k}}{(c+v)_{k} k !}
$$

of the Gaussian hypergeometric series ${ }_{2} F_{1}(a+v, b+v ; c+v ;-1)$ with $a=3 / 2, b=7 / 3$, and $c=21 / 4$. In Table 1 we have $v=0$, and in Table 2 we have $v=10$. All transformation results of this article were obtained using the floating point arithmetics of Maple 11, and the "exact" result in Tables 1 and 2 was produced by the Maple procedure hypergeom which computes generalized hypergeometric series.

The entries in the third column of Table 1 are chosen according (B.8), and the entries in columns 4 and 5 according to (B.16). Since Wynn's epsilon algorithm produces according to (B.5) Padé approximants if the input data are the partial sums of a power series, the entries in the third column can be identified with the staircase sequence $[\lfloor(n+1) / 2\rfloor /\lfloor n / 2\rfloor]$ of Padé approximants to the hypergeometric series ${ }_{2} F_{1}(a+v, b+v ; c+v ; z)$ with $z=-1$. Here, $\lfloor x\rfloor$ stands for the integral part of $x$, which is the largest integer $v$ satisfying $v \leq x$.

The asymptotic estimate (2.16) shows that the terms $(-1)^{n}(a)_{n}(b)_{n} /\left[c_{n} n\right.$ !] of the hypergeometric series in Table 1 decay in magnitude like $n^{-29 / 12}$ as $n \rightarrow \infty$. Therefore, this hypergeometric series converges, albeit quite slowly. This assessment is confirmed by the data in the second column of Table 1 . The transformation results in Table 1 indicate that all three trans- 
Table 1: Application of Wynn's epsilon algorithm and the Levin-type transformations $d_{k}^{(n)}\left(\beta, s_{n}\right)$ and $\delta_{k}^{(n)}\left(\beta, s_{n}\right)$ with $\beta=1$ to the partial sums of the slowly convergent hypergeometric series ${ }_{2} F_{1}(a+v, b+v ; c+v ;-1)$ with $a=3 / 2, b=7 / 3, c=21 / 4$, and $v=0$.

\begin{tabular}{lcccc}
\hline \hline$n$ & $s_{n}(z)$ & $\varepsilon_{2\lfloor n / 2\rfloor}^{(n-2\lfloor n / 2\rfloor)}$ & $d_{n}^{(0)}\left(1, s_{0}(z)\right)$ & $\delta_{n}^{(0)}\left(1, s_{0}(z)\right)$ \\
& Eq. (6.4) & Eq. (B.4) & Eq. (B.14) & Eq. (B.15) \\
\hline 0 & 1.00000000 & 1.000000000000000 & 1.000000000000000 & 1.000000000000000 \\
1 & 0.33333333 & 0.333333333333333 & 0.600000000000000 & 0.600000000000000 \\
2 & 0.77777778 & 0.600000000000000 & 0.596079232182969 & 0.596079232182969 \\
3 & 0.46785866 & 0.595184349134688 & 0.597361776678518 & 0.597283629053888 \\
4 & 0.69325438 & 0.597114931459132 & 0.597128156362257 & 0.597151427941970 \\
5 & 0.52349689 & 0.597142098567676 & 0.597159499152400 & 0.597156391214500 \\
6 & 0.65507045 & 0.598028964909470 & 0.597156082629105 & 0.597156376752544 \\
7 & 0.55064699 & 0.597156986187236 & 0.597156397164508 & 0.597156374043337 \\
8 & 0.63518026 & 0.597156454880736 & 0.597156372412882 & 0.597156373980610 \\
9 & 0.56559242 & 0.597156266466529 & 0.597156374069016 & 0.597156373980877 \\
10 & 0.62370437 & 0.597156373786525 & 0.597156373977124 & 0.597156373980968 \\
11 & 0.57457047 & 0.597156373530068 & 0.597156373981079 & 0.597156373980973 \\
\hline exact & & 0.597156373980973 & 0.597156373980973 & 0.597156373980973 \\
\hline \hline
\end{tabular}

formations are able to accelerate the convergence of the hypergeometric series ${ }_{2} F_{1}$ effectively. The least effective, but still very powerful accelerator is Wynn's epsilon algorithm, and the most effective transformation is the Levin-type delta transformation which is - as documented by numerous references mentioned in Appendix B - known to be highly effective in the case of both convergent and divergent alternating series.

In Table 2, only Wynn's epsilon algorithm and the delta transformation are displayed. The data in the second column show that the partial sums (6.4) with $v=10$ diverge rapidly. Nevertheless, it is possible to obtain highly accurate summation results. As in Table 1, the delta transformation was clearly more effective than Wynn's epsilon algorithm.

It is in my opinion remarkable that Levin's transformation (B.14), whose action on the partial sums (6.4) is not displayed in Table 2, turned out to be only roughly as effective as Wynn's epsilon algorithm. Under the same conditions as in Table 2, I obtained the following summation results:

$$
\begin{aligned}
& d_{16}^{(0)}\left(1, s_{0}(z)\right)=0.142894441246992 \times 10^{-2} \\
& d_{17}^{(0)}\left(1, s_{0}(z)\right)=0.142895355463039 \times 10^{-2} .
\end{aligned}
$$

In view of the in general very good reputation of Levin's sequence transformation, this comparatively weak performance is somewhat puzzling.

In the case of more complicated generalized hypergeometric series ${ }_{p+1} F_{p}$, the same general pattern was observed. If the same nonlinear sequence transformations as in Tables 1 and 
Table 2: Summation of the hypergeometric series ${ }_{2} F_{1}(a+v, b+v ; c+v ;-1)$ with $a=3 / 2$, $b=7 / 3, c=21 / 4$, and $v=10$ with the help of Wynn's epsilon algorithm and the Levin-type transformation $\delta_{k}^{(n)}\left(\beta, s_{n}\right)$ with $\beta=1$.

\begin{tabular}{lrrr}
\hline \hline & \multicolumn{1}{c}{$s_{n}(z)$} & $\varepsilon_{2\lfloor n / 2\rfloor}^{(n-2\lfloor n / 2\rfloor)}$ & \multicolumn{1}{c}{$\delta_{n}^{(0)}\left(1, s_{0}(z)\right)$} \\
& \multicolumn{1}{c}{ Eq. $(6.4)$} & Eq. (B.4) & Eq. (B.15) \\
\hline 0 & $0.10000 \times 10^{1}$ & $0.100000000000000 \times 10^{+1}$ & $0.100000000000000 \times 10^{+1}$ \\
1 & $-0.83005 \times 10^{1}$ & $-0.830054644808743 \times 10^{+1}$ & $-0.517662391110501 \times 10^{+0}$ \\
2 & $0.39395 \times 10^{2}$ & $-0.517662391110501 \times 10^{+0}$ & $0.355800262535272 \times 10^{+0}$ \\
3 & $-0.13894 \times 10^{3}$ & $0.176355932965950 \times 10^{+1}$ & $-0.979095090620413 \times 10^{-1}$ \\
4 & $0.40421 \times 10^{3}$ & $0.150567485119558 \times 10^{+0}$ & $0.156275192732603 \times 10^{-1}$ \\
5 & $-0.10245 \times 10^{4}$ & $-0.292708508243714 \times 10^{+0}$ & $0.539203120343906 \times 10^{-3}$ \\
6 & $0.23385 \times 10^{4}$ & $-0.235266825737199 \times 10^{-1}$ & $0.137225149988457 \times 10^{-2}$ \\
7 & $-0.49149 \times 10^{4}$ & $0.351057409892771 \times 10^{-1}$ & $0.145137993614918 \times 10^{-2}$ \\
8 & $0.96599 \times 10^{4}$ & $0.394766824723114 \times 10^{-2}$ & $0.142550654190640 \times 10^{-2}$ \\
9 & $-0.17957 \times 10^{5}$ & $-0.113107334634727 \times 10^{-2}$ & $0.142932832720914 \times 10^{-2}$ \\
10 & $0.31849 \times 10^{5}$ & $0.127361316374322 \times 10^{-2}$ & $0.142892054773978 \times 10^{-2}$ \\
11 & $-0.54255 \times 10^{5}$ & $0.155543251021275 \times 10^{-2}$ & $0.142895407283313 \times 10^{-2}$ \\
12 & $0.89251 \times 10^{5}$ & $0.143471035609870 \times 10^{-2}$ & $0.142895196573040 \times 10^{-2}$ \\
13 & $-0.14240 \times 10^{6}$ & $0.142504106397527 \times 10^{-2}$ & $0.142895206303027 \times 10^{-2}$ \\
14 & $0.22113 \times 10^{6}$ & $0.142883205557579 \times 10^{-2}$ & $0.142895205998921 \times 10^{-2}$ \\
15 & $-0.33524 \times 10^{6}$ & $0.142902170361815 \times 10^{-2}$ & $0.142895206004165 \times 10^{-2}$ \\
16 & $0.49740 \times 10^{6}$ & $0.142895322013589 \times 10^{-2}$ & $0.142895206004153 \times 10^{-2}$ \\
17 & $-0.72381 \times 10^{6}$ & $0.142895148436317 \times 10^{-2}$ & $0.142895206004152 \times 10^{-2}$ \\
\hline exact & & $0.142895206004152 \times 10^{-2}$ & $0.142895206004152 \times 10^{-2}$ \\
\hline \hline
\end{tabular}

2 are applied to the partial sums

$$
s_{n}=s_{n}(a, b, c, d, e, v)=\sum_{k=0}^{n}(-1)^{k} \frac{(a+v)_{k}(b+v)_{k}(c+v)_{k}}{(d+v)_{k}(e+v)_{k} k !}
$$

of the generalized hypergeometric series ${ }_{3} F_{2}(a+v, b+v, c+v ; d+v, e+v ;-1)$ with $a=3 / 2$, $b=7 / 3, c=11 / 5, d=22 / 7$, and $e=32 / 11$, we obtain for the convergent hypergeometric series with $v=0$

$$
\begin{aligned}
& \varepsilon_{12}^{(0)}=0.536266961325332, \\
& d_{12}^{(0)}\left(1, s_{0}(z)\right)=0.536266961240988, \\
& \delta_{12}^{(0)}\left(1, s_{0}(z)\right)=0.536266961240986, \\
& \text { hypergeom }=0.536266961240986,
\end{aligned}
$$


and for the divergent hypergeometric series with $v=10$

$$
\begin{aligned}
\varepsilon_{16}^{(1)} & =0.816454762672306 \times 10^{-3}, \\
d_{17}^{(0)}\left(1, s_{0}(z)\right) & =0.816459448502108 \times 10^{-3}, \\
\delta_{17}^{(0)}\left(1, s_{0}(z)\right) & =0.816458731770118 \times 10^{-3}, \\
\text { hypergeom } & =0.816458731770118 \times 10^{-3} .
\end{aligned}
$$

In the case of the partial sums

$$
s_{n}=s_{n}(a, b, c, d, e, f, g v)=\sum_{k=0}^{n}(-1)^{k} \frac{(a+v)_{k}(b+v)_{k}(c+v)_{k}(d+v)_{k}}{(e+v)_{k}(f+v)_{k}(g+v)_{k} k !}
$$

of the generalized hypergeometric series ${ }_{4} F_{3}(a+v, b+v, c+v, d+v ; e+v, f+v, g+v ;-1)$ with $a=3 / 2, b=7 / 3, c=11 / 5, d=16 / 17, e=18 / 19, f=22 / 7$, and $g=32 / 11$, we obtain for the convergent hypergeometric series with $v=0$

$$
\begin{aligned}
& \varepsilon_{12}^{(0)}=0.538509188429164, \\
& d_{12}^{(0)}\left(1, s_{0}(z)\right)=0.538509188330837, \\
& \delta_{12}^{(0)}\left(1, s_{0}(z)\right)=0.538509188330835, \\
& \text { hypergeom }=0.538509188330835,
\end{aligned}
$$

and for the divergent hypergeometric series with $v=10$

$$
\begin{aligned}
\varepsilon_{16}^{(1)} & =0.819691553980977 \times 10^{-3}, \\
d_{17}^{(0)}\left(1, s_{0}(z)\right) & =0.819696193819277 \times 10^{-3}, \\
\delta_{17}^{(0)}\left(1, s_{0}(z)\right) & =0.819695479036364 \times 10^{-3}, \\
\text { hypergeom } & =0.819695479036364 \times 10^{-3} .
\end{aligned}
$$

It was emphasized in Section 5 that the inner $\mu$ series in (3.14) converge if the coefficients $\lambda_{n}^{(\alpha)}$ of a Laguerre series decay exponentially as $n \rightarrow \infty$. Accordingly, summation techniques are not needed in this case. Nevertheless, nonlinear sequence transformations can be extremely useful even in the case of exponentially decaying Laguerre series coefficients. Let us for example assume that the coefficients $\lambda_{n}^{(\alpha)}$ of a Laguerre series satisfy

$$
\lambda_{n}^{(\alpha)}=t^{n} \ell_{n}^{(n)}
$$

and that it is not possible to derive a closed form expression for the inner $\mu$ series in (3.14). Thus, the the inner $\mu$ series have to be evaluated numerically.

Let us now also assume that the coefficients $\ell_{n}^{(n)}$ increase like a fixed power of $n$ as $n \rightarrow \infty$. In spite of this unfavorable behavior, the inner $\mu$ series converge as long as $|t|<1$. However, convergence can become prohibitively slow if $|t|$ is only slightly smaller than one. The convergence problems are particularly severe if the terms in inner $\mu$ series ultimately have the same sign because then the $\mu$ series do not converge for $t=1$ and are also not summable. But again, nonlinear sequence transformations can be extremely useful to speed up the convergence of such a monotone series with (very) slowly decaying terms (see for example [85] or [27, Section 2.2.6] and references therein). 
As documented by the recent books by Cuyt, Brevik Petersen, Verdonk, Waadeland, and Jones [36] and by Gil, Segura, and Temme [48], or by a review by Temme [128], there is currently a lot of work being done on the efficient and reliable evaluation of special functions. As I had shown in several articles [84, 85, 136, 137, 139, 140, 143, 147, 148, 155, 162], nonlinear sequence transformations can be extremely useful in this respect.

\section{Guseinov's Rearranged One-Range Addition Theorems}

The analyticity of Laguerre series is a problem of classical analysis, but I became interested in this mathematical topic because of some open questions in molecular electronic structure theory. During the work for my forthcoming review on addition theorems [154], I came across some articles by Guseinov [53, 54, 56] who had constructed one-range addition theorems for Slater-type functions [117, 118]. In unnormalized form, Slater-type functions, which play a major role as basis functions in atomic and molecular electronic structure calculations, are defined as follows:

$$
\chi_{N, L}^{M}(\beta, \mathbf{r})=(\beta r)^{N-L-1} \mathrm{e}^{-\beta r} \mathscr{Y}_{L}^{M}(\beta \boldsymbol{r}) .
$$

Here, $\boldsymbol{r} \in \mathbb{R}^{3}, \mathscr{Y}_{L}^{M}(\beta \boldsymbol{r})=(\beta r)^{L} Y_{L}^{M}(\theta, \phi)$ is a regular solid harmonic and $Y_{L}^{M}(\theta, \phi)$ is a (surface) spherical harmonic, $\beta>0$ is a scaling parameter, $N \in \mathbb{R} \backslash \mathbb{N}$ is a kind of generalized principal quantum number which is often, but not always a positive integer $\geq L+1$, and $L$ and $M$ are the usual (orbital) angular momentum quantum numbers.

Let us assume that $\left\{\varphi_{n, \ell}^{m}(\boldsymbol{r})\right\}_{n, \ell, m}$ is a complete and orthonormal function set in the Hilbert space

$$
L^{2}\left(\mathbb{R}^{3}\right)=\left\{f:\left.\mathbb{R}^{3} \rightarrow \mathbb{C}\left|\int\right| f(\boldsymbol{r})\right|^{2} \mathrm{~d}^{3} \boldsymbol{r}<\infty\right\}
$$

of functions that are square integrable with respect to an integration over the whole $\mathbb{R}^{3}$. Since any $f \in L^{2}\left(\mathbb{R}^{3}\right)$ can be expanded in terms of the complete and orthonormal functions $\left\{\varphi_{n, \ell}^{m}(\boldsymbol{r})\right\}_{n, \ell, m}$, a one-range addition theorem for $f\left(\boldsymbol{r} \pm \boldsymbol{r}^{\prime}\right)$ can be formulated as follows:

$$
\begin{aligned}
f\left(\boldsymbol{r} \pm \boldsymbol{r}^{\prime}\right) & =\sum_{n \ell m} C_{n, \ell}^{m}\left(f ; \pm \boldsymbol{r}^{\prime}\right) \varphi_{n, \ell}^{m}(\boldsymbol{r}), \\
C_{n, \ell}^{m}\left(f ; \pm \boldsymbol{r}^{\prime}\right) & =\int\left[\varphi_{n, \ell}^{m}(\boldsymbol{r})\right]^{*} f\left(\boldsymbol{r} \pm \boldsymbol{r}^{\prime}\right) \mathrm{d}^{3} \boldsymbol{r} .
\end{aligned}
$$

The expansion (7.3), which converges in the mean with respect to the norm of the Hilbert space $L^{2}\left(\mathbb{R}^{3}\right)$, is a one-range addition theorem since the variables $\boldsymbol{r}$ and $\boldsymbol{r}^{\prime}$ are completely separated: The dependence on $\boldsymbol{r}$ is entirely contained in the functions $\varphi_{n, \ell}^{m}(\boldsymbol{r})$, whereas $\boldsymbol{r}^{\prime}$ occurs only in the expansion coefficients $C_{n, \ell}^{m}\left(f ; \pm \boldsymbol{r}^{\prime}\right)$ which are overlap or convolution-type integrals.

One-range addition theorems of the kind of (7.3) were constructed by Filter and Steinborn [45, Eqs. (5.11) and (5.12)] and later applied by Kranz and Steinborn [98] and by Trivedi and Steinborn [132]. An alternative derivation of these addition theorems based on Fourier transformation combined with weakly convergent expansions of the plane wave $\exp ( \pm \mathrm{i} \boldsymbol{p} \cdot \boldsymbol{r})$ with $\boldsymbol{p}, \boldsymbol{r} \in \mathbb{R}^{3}$ was presented in [135, Section VII].

As discussed in [151, Section 3]), it is also possible to formulate one-range addition theorems that converge with respect to the norm of a weighted Hilbert space

$$
L_{w}^{2}\left(\mathbb{R}^{3}\right)=\left\{f:\left.\mathbb{R}^{3} \rightarrow \mathbb{C}\left|\int w(\boldsymbol{r})\right| f(\boldsymbol{r})\right|^{2} \mathrm{~d}^{3} \boldsymbol{r}<\infty\right\},
$$


where $w(\boldsymbol{r}) \neq 1$ is a suitable positive weight function. If we assume that $f \in L_{w}^{2}\left(\mathbb{R}^{3}\right)$ and that the functions $\left\{\psi_{n, \ell}^{m}(\boldsymbol{r})\right\}_{n, \ell, m}$ are complete and orthonormal in $L_{w}^{2}\left(\mathbb{R}^{3}\right)$, then we obtain the following one-range addition theorem [151, Eq. (3.6)]):

$$
\begin{aligned}
f\left(\boldsymbol{r} \pm \boldsymbol{r}^{\prime}\right) & =\sum_{n \ell m} \mathbf{C}_{n, \ell}^{m}\left(f, w ; \pm \boldsymbol{r}^{\prime}\right) \psi_{n, \ell}^{m}(\boldsymbol{r}), \\
\mathbf{C}_{n, \ell}^{m}\left(f, w ; \pm \boldsymbol{r}^{\prime}\right) & =\int\left[\psi_{n, \ell}^{m}(\boldsymbol{r})\right]^{*} w(\boldsymbol{r}) f\left(\boldsymbol{r} \pm \boldsymbol{r}^{\prime}\right) \mathrm{d}^{3} \boldsymbol{r}
\end{aligned}
$$

A one-range addition theorem for a function $f: \mathbb{R}^{3} \rightarrow \mathbb{C}$ is a mapping $\mathbb{R}^{3} \times \mathbb{R}^{3} \rightarrow \mathbb{C}$. Compared to the better known two-range addition theorems like the so-called Laplace expansion of the Coulomb or Newton potential $1 / r$, which possesses a characteristic two-range form (see for example [151, Eq. (1.2)]), one-range addition theorems have the highly advantageous feature that they provide a unique infinite series representation of $f\left(\boldsymbol{r} \pm \boldsymbol{r}^{\prime}\right)$ with separated variables $\boldsymbol{r}$ and $\boldsymbol{r}^{\prime}$ that is valid for the whole argument set $\mathbb{R}^{3} \times \mathbb{R}^{3}$. Further properties of addition theorems in general and of one-range addition theorems in particular will be discussed in my forthcoming review [154].

In his one-range addition theorems, Guseinov used as a complete and orthonormal function set the following functions [55, Eq. (1)], which - if the mathematical notation for the generalized Laguerre polynomials is used - can be expressed as follows [151, Eq. (4.16)]:

$$
\begin{gathered}
{ }_{k} \Psi_{n, \ell}^{m}(\gamma, \boldsymbol{r})=\left[\frac{(2 \gamma)^{k+3}(n-\ell-1) !}{(n+\ell+k+1) !}\right]^{1 / 2} \mathrm{e}^{-\gamma r} L_{n-\ell-1}^{(2 \ell+k+2)}(2 \gamma r) \mathscr{Y}_{\ell}^{m}(2 \gamma \boldsymbol{r}), \\
n \in \mathbb{N}, \quad k=-1,0,1,2, \ldots, \quad \gamma>0 .
\end{gathered}
$$

As discussed in the text following [151, Eq. (4.20)], Guseinov's functions (7.6) can - depending on the value of $k=-1,0,1,2, \ldots$ - reproduce several other physically relevant complete and orthonormal function sets.

Guseinov's functions are orthonormal with respect to the weight function $w(\boldsymbol{r})=r^{k}$ (compare also [56, Eq. (4)]):

$$
\int\left[{ }_{k} \Psi_{n, \ell}^{m}(\gamma, \boldsymbol{r})\right]^{*} r_{k}^{k} \Psi_{n^{\prime}, \ell^{\prime}}^{m^{\prime}}(\gamma, \boldsymbol{r}) \mathrm{d}^{3} \boldsymbol{r}=\delta_{n n^{\prime}} \boldsymbol{\delta}_{\ell \ell^{\prime}} \boldsymbol{\delta}_{m m^{\prime}}
$$

Accordingly, Guseinov's functions are complete and orthonormal in the weighted Hilbert space

$$
L_{r^{k}}^{2}\left(\mathbb{R}^{3}\right)=\left\{f:\left.\mathbb{R}^{3} \rightarrow \mathbb{C}\left|\int r^{k}\right| f(\boldsymbol{r})\right|^{2} \mathrm{~d}^{3} \boldsymbol{r}<\infty\right\}, \quad k=-1,1,2, \ldots
$$

For $k=0$, we retrieve the Hilbert space $L^{2}\left(\mathbb{R}^{3}\right)$ of square integrable functions defined by (7.2).

As long as the principal quantum number $N$ is not too negative, a Slater-type function $\chi_{N, L}^{M}(\beta, r)$ is for a fixed value of $k$ an element of the weighted Hilbert space $L_{r^{k}}^{2}\left(\mathbb{R}^{3}\right)$. In this case, Guseinov's approach [53, 54, 56], who constructed one-range addition theorems by expanding $\chi_{N, L}^{M}\left(\beta, r \pm \boldsymbol{r}^{\prime}\right)$ in terms of his complete and orthonormal functions $\left\{{ }_{k} \Psi_{n, \ell}^{m}(\gamma, \boldsymbol{r})\right\}_{n, \ell, m}$ with in general different scaling parameters $\beta \neq \gamma>0$, is mathematically sound. For fixed $k=-1,0,1,2, \ldots$, Guseinov constructed expansions that converge in the mean with respect to the norm of $L_{r^{k}}^{2}\left(\mathbb{R}^{3}\right)$.

However, Guseinov replaced in his one-range addition theorems his complete and orthonormal functions ${ }_{k} \Psi_{n, \ell}^{m}(\beta, r)$ by nonorthogonal Slater-type functions with integral princi- 
pal quantum numbers via [151, Eq. (6.4)]

$$
\begin{aligned}
{ }_{k} \Psi_{n, \ell}^{m}(\beta, \boldsymbol{r})=2^{\ell} & {\left[\frac{(2 \beta)^{k+3}(n+\ell+k+1) !}{(n-\ell-1) !}\right]^{1 / 2} } \\
& \times \sum_{v=0}^{n-\ell-1} \frac{(-n+\ell+1)_{v} 2^{v}}{(2 \ell+k+v+2) ! v !} \chi_{v+\ell+1, \ell}^{m}(\beta, \boldsymbol{r}) .
\end{aligned}
$$

This is still legitimate. However, Guseinov also rearranged the order of summations of the resulting expansions. In this way, Guseinov formally constructed expansions of Slater-type functions $\chi_{N, L}^{M}\left(\beta, \mathbf{r} \pm \mathbf{r}^{\prime}\right)$ with in general nonintegral principal quantum numbers $N \in \mathbb{R} \backslash \mathbb{N}$ in terms of Slater-type functions $\chi_{n, \ell}^{m}(\beta, \mathbf{r})$ with integral principal quantum numbers $n \in \mathbb{N}$ located at a different center (see also [151, Section 6]).

Slater-type functions are complete in all Hilbert spaces, which Guseinov implicitly used (for an explicit proof, see [91, Section 4]), but not orthogonal. Thus, Guseinov's approach corresponds to the transformation of an expansion in terms of a complete and orthogonal function set to an expansion in terms a of a complete, but nonorthogonal function set.

Unfortunately, the completeness of a nonorthogonal function set in a Hilbert space does not suffice to guarantee that an essentially arbitrary element of this Hilbert space can be expanded in terms of this function set (the nonanalyticity of certain Laguerre series discussed in this article is just another confirmation of a much more general fact). This insufficiency is well documented both in the mathematical literature (see for example [38, Theorem 10 on p. 54] or [79, Section 1.4]) as well as in the literature on electronic structure calculations [88, 89, 90, 91, 92, 93]), but nevertheless frequently overlooked. Horrifying examples of nonorthogonal expansions with pathological properties can be found in [89, Section III.I].

Consequently, it is not at all obvious whether the mathematical manipulations, that produced Guseinov's rearranged one-range addition theorems, are legitimate and lead to expansions that are mathematically meaningful. This has to be checked. So far, Guseinov has categorically denied that there might be any problem with the legitimacy of his rearrangements [71, pp. 8 and 23 - 24].

Since Guseinov's original addition theorems are expansions in terms of generalized Laguerre polynomials, and since the radial parts of Slater-type functions are after the cancellation of common exponentials nothing but powers, the results of this article about the analyticity of Laguerre series could in principle be used to investigate whether Guseinov's rearrangements are legitimate. For that purpose, it would be necessary to determine the decay rates and the sign patterns of the coefficients of the generalized Laguerre polynomials occurring in Guseinov's original addition theorems.

Unfortunately, one-range addition theorems for Slater-type functions are fairly complicated mathematical objects, and the coefficients of the generalized Laguerre polynomials are according to (7.5) essentially three-dimensional overlap integrals. Thus, we would be confronted with enormous and possibly even unsurmountable technical problems if we try to analyze the decay rates and sign patters of the coefficients of these Laguerre series.

Fortunately, some insight can be gained by analyzing not the comparatively complicated one-range addition theorems, but their much simpler one-center limits. These are also expansions in terms of generalized Laguerre polynomials, albeit with much simpler coefficients. It will become clear later that this approach cannot answer all questions in interest. Nevertheless, it is better than nothing since we can obtain at least some nontrivial answers.

Let us now consider the one-center expansion of a Slater-type function $\chi_{N, L}^{M}(\beta, \mathbf{r})$ with in general nonintegral principal quantum numbers $N \in \mathbb{R} \backslash \mathbb{N}$ in terms of Guseinov's functions 
${ }_{k} \Psi_{n, \ell}^{m}(\beta, r)$ with equal scaling parameters $\beta>0$ [152, Eq. (5.7)]:

$$
\begin{aligned}
& \chi_{N, L}^{M}(\beta, r)=\frac{(2 \gamma)^{-(k+3) / 2}}{2^{N-1}} \Gamma(N+L+k+2) \\
& \times \sum_{v=0}^{\infty} \frac{(-N+L+1)_{v}}{[(v+2 L+k+2) ! v !]^{1 / 2}}{ }_{k} \Psi_{v+L+1, L}^{M}(\beta, r), \\
& N \in \mathbb{R} \backslash \mathbb{N}, \quad \beta>0, \quad k=-1,0,1,2, \ldots
\end{aligned}
$$

If $N \in \mathbb{N}$ and $N \geq L+1$, the infinite series on the right-hand side terminates because of the Pochhammer symbol $(-N+L+1)_{v}$.

The expansion (7.10), which corresponds to the one-center limit $\boldsymbol{r}^{\prime}=\mathbf{0}$ of Guseinov's one-range addition theorem for $\chi_{N, L}^{M}\left(\beta, \mathbf{r} \pm \boldsymbol{r}^{\prime}\right)$ with equal scaling parameters, is a special case of the Laguerre series (3.3) for $z^{\rho}$ with in general nonintegral $\rho \in \mathbb{R} \backslash \mathbb{N}_{0}$. As discussed in Section 3, a rearrangement of the Laguerre series (3.3) for $z^{\rho}$ is legitimate if and only if $\rho$ is a nonnegative integer, i.e., if $\rho=m \in \mathbb{N}_{0}$. If $\rho \notin \mathbb{N}_{0}, z^{\rho}$ is not analytic at $z=0$ and we formally obtain the power series (3.7), which is not a mathematically meaningful object since an infinite number of its power series coefficients are according to (3.9) infinite in magnitude.

Thus, in the case of equal scaling parameters $\beta>0$, the one-center limit $\boldsymbol{r}^{\prime}=\mathbf{0}$ of Guseinov's rearranged addition theorem for Slater-type functions $\chi_{N, L}^{M}\left(\beta, r \pm \boldsymbol{r}^{\prime}\right)$ does not exist if the principal quantum number $N$ is nonintegral, i.e., if $N \in \mathbb{R} \backslash \mathbb{N}$.

Let us now consider the one-center expansion of a Slater-type function $\chi_{N . L}^{M}(\beta, \mathbf{r})$ with in general nonintegral principal quantum numbers $N \in \mathbb{R} \backslash \mathbb{N}$ in terms of Guseinov's functions ${ }_{k} \Psi_{n, \ell}^{m}(\gamma, \boldsymbol{r})$ with different scaling parameters $\beta \neq \gamma>0$ [152, Eq. (5.9)]:

$$
\begin{aligned}
\chi_{N, L}^{M}(\beta, r)=\frac{(2 \gamma)^{L+(k+3) / 2} \beta^{N-1}}{[\beta+\gamma]^{N+L+k+2}} \frac{\Gamma(N+L+k+2)}{(2 L+k+2) !} \\
\times \sum_{v=0}^{\infty}\left[\frac{(v+2 L+k+2) !}{v !}\right]_{k}^{1 / 2} \Psi_{v+L+1, L}^{M}(\gamma, \boldsymbol{r}) \\
\quad \times{ }_{2} F_{1}\left(-v, N+L+k+2 ; 2 L+k+3 ; \frac{2 \gamma}{\beta+\gamma}\right) .
\end{aligned}
$$

The expansion (7.11), which corresponds to the one-center limit $\boldsymbol{r}^{\prime}=\mathbf{0}$ of Guseinov's onerange addition theorem for $\chi_{N, L}^{M}\left(\beta, \mathbf{r} \pm \boldsymbol{r}^{\prime}\right)$ with different scaling parameters, is a special case of the following Laguerre series [151, Eq. (6.12)]:

$$
\begin{aligned}
z^{\rho} \mathrm{e}^{u z}=(1-u)^{-\alpha-\rho-1} & \frac{\Gamma(\alpha+\rho+1)}{\Gamma(\alpha+1)} \\
& \times \sum_{n=0}^{\infty}{ }_{2} F_{1}\left(-n, \alpha+\rho+1 ; \alpha+1 ; \frac{1}{1-u}\right) L_{n}^{(\alpha)}(z), \\
& \rho \in \mathbb{R} \backslash \mathbb{N}_{0}, \quad \operatorname{Re}(\rho+\alpha)>-1, \quad u \in(-\infty, 1 / 2) .
\end{aligned}
$$

This expansion can be derived with the help of (3.2). The condition $-\infty<u<1 / 2$ is necessary to guarantee its convergence in the mean with respect to the norm (2.6) of the weighted Hilbert space $L_{z^{\alpha} \mathrm{e}^{-z}}^{2}([0, \infty))$. For $u=0$, the terminating Gaussian hypergeometric series ${ }_{2} F_{1}$ can be expressed in closed form with the help of Gauss' summation theorem [102, p. 40], and (7.12) simplifies to give (3.3).

If we insert the explicit expression (2.1) of the generalized Laguerre polynomials into (7.12) and interchange the order of summations, we also obtain a formal power series in $z$.

Ernst Joachim Weniger: On the Analyticity of Laguerre Series 
Unfortunately, an analysis of the resulting power series becomes very difficult because of the terminating Gaussian hypergeometric series ${ }_{2} F_{1}$ in (7.12). An analysis of the behavior of this ${ }_{2} F_{1}$ as $n \rightarrow \infty$ would most likely be a nontrivial research project in its own right. However, we can argue that $z^{\rho} \exp (u z)$ is only analytic at $z=0$ if $\rho=m \in \mathbb{N}_{0}$, yielding the expansion $z^{m} \exp (u z)=\sum_{n=0}^{\infty} u^{n} z^{m+n} / n$ !. If $\rho$ is nonintegral, a power series expansion of $z^{\rho} \exp (u z)$ about $z=0$ does not exist.

Thus, also for different scaling parameters $\beta \neq \gamma$, the one-center limit $\boldsymbol{r}^{\prime}=\mathbf{0}$ of the rearranged addition theorems for $\chi_{N, L}^{M}\left(\beta, r \pm \boldsymbol{r}^{\prime}\right)$ does not exist if the principal quantum number $N$ is nonintegral, i.e., if $N \in \mathbb{R} \backslash \mathbb{N}$.

These observations are quite consequential: Rearranged one-range addition theorems for Slater-type functions $\chi_{N, L}^{M}\left(\beta, r \pm \boldsymbol{r}^{\prime}\right)$ with nonintegral principal quantum numbers $N \neq 1,2, \ldots$ play a central role in numerous articles by Guseinov and coworkers on one-range addition theorems [55, 57, 58, 59, 60, 61, 62, 63, 64, 65, 66, 67, 68, 69, 70, 72, 73, 74, 75, 76, 77.

By analyzing the rearrangement of the Laguerre series of the comparatively simple functions $z^{\rho}$ and $z^{\rho} \exp (u z)$, we could arrive at some conclusions about the legitimacy of Guseinov's approach. Nevertheless, some interesting questions are still open. For example, the nonanalyticity arguments presented here allow no conclusions about the validity of Guseinov's rearrangements in the case of Slater-type functions with integral principal quantum numbers.

Another interesting but open question is whether Guseinov's rearrangements produce in the case of nonintegral principal quantum numbers one-range addition theorems that are invalid for the whole argument set $\mathbb{R}^{3} \times \mathbb{R}^{3}$, or whether only the one-center limits of these addition theorems are invalid. This is a practically very relevant question. If only the onecenter limits are invalid, then it would be conceivable that Guseinov's rearranged one-range addition theorems might be mathematically meaningful or possibly even numerically useful in a restricted sense as approximations, although they do not exist for the whole argument set $\mathbb{R}^{3} \times \mathbb{R}^{3}$. This remains to be investigated. But the burden of proof lies in all cases with Guseinov.

\section{Summary and Conclusions}

The generalized Laguerre polynomials belong to the so-called classical orthogonal polynomials of mathematical physics, and they are characterized by the orthogonality relationship (2.4) that involves an integration over the semi-infinite positive real axis. Accordingly, generalized Laguerre polynomials can be used for the representation of functions on unbounded domains, and in particular also for the representation of the radial parts of functions $f: \mathbb{R}^{3} \rightarrow \mathbb{C}$ expressed in terms of spherical polar coordinates.

It is generally accepted that expansions in terms of orthogonal polynomials have many highly advantageous features. However, there is one undeniable drawback: Normally, expansions in terms of orthogonal polynomials converge in the mean with respect to the norm of the corresponding Hilbert space, but not necessarily pointwise. Accordingly, orthogonal expansions are not necessarily the best choice if the local properties of a function matter. As documented by the popularity of Padé approximants, power series are also not free of weaknesses, but at least in the vicinity of the expansion point, power series are normally very convenient and very useful for an accurate description of the local properties of a function. Therefore, the construction of power series expansions for functions defined by orthogonal expansions should be of principal interest.

This article describes a complementary treatment of Laguerre series of the type of (1.1).

Ernst Joachim Weniger: On the Analyticity of Laguerre Series 
Normally, one starts from a known function $f(z)$ belonging to the weighted Hilbert space $L_{z^{\alpha} \mathrm{e}^{-z}}^{2}([0, \infty))$ defined by (2.7), and one tries to determine the coefficients $\lambda_{n}^{(\alpha)}$ via (1.1b) by exploiting the orthogonality of the generalized Laguerre polynomials.

In this article, it is instead assumed that only the Laguerre series coefficients $\lambda_{n}^{(\alpha)}$ are known, either in the form of explicit expressions or numerically, but not the function $f(z)$. With the help of the transformation formula (3.14), it is then possible to construct a formal power series expansion of the unknown function represented by the Laguerre series.

This approach does not guarantee success since there are many functions which belong to the Hilbert space $L_{z^{\alpha} \mathrm{e}^{-z}}^{2}([0, \infty))$ but which are not analytic at the origin. A simple example of such a nonanalytic function possessing a Laguerre series is the power function $z^{\rho}$ with nonintegral $\rho \in \mathbb{R} \backslash \mathbb{N}_{0}$. As discussed in Section 3, it is possible to construct a formal power series for $z^{\rho}$ from its Laguerre series (3.3), but the resulting power series is mathematically meaningless since it contains infinitely many power series coefficients that are infinite in magnitude.

Thus, the key question is whether the power series for the unknown function obtained via (3.14) is mathematically meaningless, or whether this power series represents an analytic function in the sense of complex analysis. It seems that this question has not been treated properly in the literature yet. I am only aware of short remarks by Gottlieb and Orszag [49. p. 42] and by Doha [41, p. 5452], respectively, who had stated that a Laguerre series of the type of (1.1) converges faster than algebraically if the function under consideration is analytic at the origin. But this statement is imprecise and ignores the pivotal role played by divergent, but summable inner $\mu$ series in (3.14). Some general aspects of the summation of divergent series are reviewed in Appendix $\mathrm{A}$

By analyzing the convergence properties of the inner $\mu$ series in (3.14), some simple sufficient conditions can be formulated which guarantee that the resulting power series is mathematically meaningful and represents an analytic function.

As discussed in Section 5 the most benign situation occurs if the Laguerre series coefficients $\lambda_{n}^{(\alpha)}$ decay exponentially or even factorially as $n \rightarrow \infty$. Then, the inner $\mu$ series in (3.14) converge and the resulting power series is mathematically meaningful and represents an analytic function. In this way, numerous generating functions for the generalized Laguerre polynomials can be rederived easily.

As discussed in Section 4, a much more interesting situation occurs if the coefficients $\lambda_{n}^{(\alpha)}$ decay algebraically in magnitude as $n \rightarrow \infty$. If algebraically decaying coefficients $\lambda_{n}^{(\alpha)}$ ultimately have the same sign, the inner $\mu$ series in (3.14) diverge. This alone wound not necessarily be such a bad thing, but it is not possible to sum these divergent series to something finite. Accordingly, the transformation formula (3.14) leads to a power series expansion having infinitely many series coefficients that are infinite in magnitude. This simply means that a power series of the type of (1.3) does not exist because the function under consideration is not analytic at the origin. An example is the Laguerre series (3.3) for $z^{\rho}$ : Its series coefficients decay algebraically in magnitude and ultimately all have the same sign. This observation suffices to show once more that $z^{\rho}$ is not analytic at the origin if $\rho \in \mathbb{R} \backslash \mathbb{N}_{0}$.

The situation changes radically if the coefficients $\lambda_{n}^{(\alpha)}$ decay algebraically in magnitude as $n \rightarrow \infty$, but ultimately have strictly alternating signs. Then, the inner $\mu$ series in (3.14) still do not converge, but now summation techniques can be used to associate finite values to these divergent alternating series. In such a case, the formal power series obtained via (3.14) is mathematically meaningful and represents an analytic function.

For example, the additional sign factor $(-1)^{n}$ introduced into the Laguerre series (3.3) for $z^{\rho}$ yields the Laguerre series (4.7). The application of (3.14) to 4.7) leads to divergent 
inner $\mu$ series which correspond to divergent hypergeometric series ${ }_{1} F_{0}$. But these series can be summed by analytic continuation since they are special cases of the binomial series (3.8). Thus, the transformation formula (3.14) ultimately produces according to (4.12) a confluent hypergeometric series ${ }_{1} F_{1}$, which is an analytic function in every neighborhood of the origin and which is also a known generating function of the generalized Laguerre polynomials.

The summability approach pursued in Section 4 can also be used in the case of Laguerre series with more complicated coefficients. An example is the Laguerre series (4.21). The application of the transformation formula (3.14) leads to divergent, but summable $\mu$ series that can be expressed as a divergent Gaussian hypergeometric series ${ }_{2} F_{1}$. But again, the summation of this divergent series is almost trivially simple because many convenient analytic continuation formulas for $\mathrm{a}_{2} F_{1}$ are known.

Thus, the summation of a divergent inner $\mu$ series can be accomplished by explicit analytic continuation formulas if it can be expressed either as a divergent binomial series ${ }_{1} F_{0}$ or as a divergent Gaussian hypergeometric series ${ }_{2} F_{1}$. Unfortunately, this is no longer possible if the $\mu$ series corresponds to a divergent generalized hypergeometric series ${ }_{p+1} F_{p}$ with $p \geq 2$. As discussed in Section 6 analytic continuation formulas for more complicated generalized hypergeometric series are either not known at all or at least much more complicated than the corresponding formulas for Gaussian hypergeometric series.

Thus, we can only hope to find convenient analytical expressions for power series coefficients $\gamma_{n}$ if the corresponding algebraically decaying and ultimately strictly alternating Laguerre series coefficients $\lambda_{n}^{(\alpha)}$ possess a very simple structure. But this is a typical limitation of all analytical manipulations. As a viable alternative, we can try to use instead techniques that accomplish a summation of divergent inner $\mu$ series by purely numerical means. Such an approach has the additional advantage that it can be applied if only the numerical values of a finite set of Laguerre series coefficients $\lambda_{n}^{(\alpha)}$ are available.

In Section 6, it is shown that certain nonlinear sequence transformations, whose properties are reviewed in Appendix B, are indeed able to sums divergent alternating hypergeometric series ${ }_{p+1} F_{p}$ with $p \geq 1$ quite effectively. It may be surprising for nonspecialists that the best summation results were not obtained by Wynn's celebrated epsilon algorithm (B.4), which produces Padé approximants if the input data are the partial sums of a power series, but by the Levin-type transformation (B.15).

In Section 7 the legitimacy of the rearrangement of Guseinov's one-range addition theorems for Slater-type functions with in general nonintegral principal quantum numbers is analyzed. Originally, Guseinov [53, 54, 56] had derived addition theorems for Slater-type functions by expanding them in terms of the complete and orthonormal functions (7.6) whose radial parts consist of generalized Laguerre polynomials. In the next step, Guseinov replaced the functions (7.6) according to (7.9) by Slater-type functions with integral principal quantum numbers, which are complete, but not orthogonal, and he also rearranged the order of the resulting summations. In this way, Guseinov essentially replaced expansions in terms of generalized Laguerre polynomials by power series expansions. Thus, the results of this article can in principle be used to check the legitimacy of Guseinov's manipulations.

Since, however, one-range addition theorems for Slater-type functions are fairly complicated mathematical objects, the necessary determination of the decay rates and the sign patterns of the coefficients of the generalized Laguerre polynomials is very difficult. Fortunately, one can gain at least some insight by analyzing not the complicated addition theorems, but their much simpler one-center limits. In this way, it can for instance be shown that Guseinov's rearranged addition theorems for Slater-type functions with nonintegral principal quantum numbers do not exist for the whole argument range $\mathbb{R}^{3} \times \mathbb{R}^{3}$.

Ernst Joachim Weniger: On the Analyticity of Laguerre Series 


\section{A Divergent Series}

Divergent series have been a highly controversial topic that played a major role in the development of mathematical analysis (see for example the article by Burkhardt [26] or the very recent book by Ferraro [44]), and even now there is still a lot of active research on divergent series going on (see for example the recent monographs by Balser [4, 5], Boutet de Monvel [17], Candelpergher, Nosmas, and Pham [28], and Sternin and Shatalov [123], or the review by Delabaere and Pham [39]).

As for instance discussed in articles by Barbeau [6], Barbeau and Leah [7], Kozlov [97], and Varadarajan [112], already Euler had frequently used divergent series. Later, when the concept of convergence was better understood, Euler's admittedly somewhat informal treatment of divergent series was criticized, and a strong tendency emerged to ban divergent series completely from the realm of rigorous mathematics. This criticism culminated in Abel's famous quotation from the year 1828, which very well expressed the prevalent attitude of most mathematicians during a large part of the nineteenth century and which can for instance be found in Littlewood's preface of Hardy's posthumously published classic on divergent series [78]:

\section{Divergent series are the invention of the devil, and it is shameful to base on them any demonstration whatsoever.}

Although this disdain of divergent series was a temporary phenomenon of the nineteenth century, its consequences are nevertheless felt today. As emphasized by Suslov [124, p. 1191], the standard university curricula in mathematical analysis were formulated in the middle of the nineteenth century. But this was a time when divergent series were wrongly considered to be essentially an aberration of the pre-rigorous mathematical past. As a consequence, divergent series and their summation are not part of the regular training of mathematicians and theoretical physicists, which in my opinion is totally inappropriate. In this context, a short article by Rubel [110] may also be of interest.

At the end of the nineteenth century it was clear that the attempts of mathematical orthodoxy to reject divergent series as unfounded had failed. Firstly, divergent series turned out to be too useful to be abandoned. For example, many special functions possess so-called asymptotic expansions which normally are factorially divergent inverse power series. In spite of their divergence, suitably truncated asymptotic series can provide excellent approximations at least for sufficiently large arguments.

Secondly, the work of mathematicians like

- Poincaré, whose work in astronomy inspired him to formulate a mathematically rigorous theory of asymptotic series that typically diverge factorially,

- Borel, who showed that factorially divergent series can be summed via Laplace-type integral representations,

- Padé, who introduced his celebrated rational approximants that are often able to sum divergent power series,

- Stieltjes, who showed that certain divergent series can be identified with continued fractions,

ultimately led in the later part of the nineteenth century to a theory which made it possible to use divergent series in a mathematically rigorous way. Even more important from a practical point of view is that their work showed that divergent series can actually be used for computational purposes if they are combined with suitable summation techniques. 
In physics, divergent series are now indispensable. It is generally accepted that perturbation theory is the most important systematic approximation procedure in theoretical physics. But already in 1952, Dyson [42] had argued that perturbation expansions in quantum electrodynamics must diverge factorially, and since the seminal work of Bender and Wu on the perturbation expansions of anharmonic oscillators [9, 10] it has been clear that quantum mechanical perturbation theory produces almost by default factorially divergent perturbation expansions. A good source on divergent perturbation expansions in quantum mechanics and in higher field theories is the book edited by Le Guillou and Zinn-Justin [99] where many of the relevant articles are reprinted.

Even if we agree that divergent series are now indispensable in physics, we nevertheless cannot expect that rigorously minded mathematicians are necessarily satisfied with the way divergent series are typically used in physics. Quite instructive is the following remark by Haldane from the year 1941, which is quoted in a book by Körner [96, p. 426]:

Cambridge is full of mathematicians who have been so corrupted by quantum mechanics that they use series which are clearly divergent, and not even proved to be summable.

I think that the criticism, which is implicit in Haldane's remark, cannot be dismissed lightly. Let us for instance assume that we want to express a physical quantity by a divergent series, which for example may be a factorially divergent Rayleigh-Schrödinger perturbation expansion. Since divergent series are a priori mathematically undefined, we have to show that it is indeed possible to associate something finite - the value of the physical quantity - to the divergent series in a mathematically meaningful way, or to put it differently, we have to show that the divergent series is summable to the correct finite result by an appropriate summation method.

Unfortunately, it is often extremely difficult to prove this rigorously. In such a situation, physicists tend to rely on their intuition and do not bother to try to formulate difficult proofs. Quite often, such a pragmatic approach is remarkably successful, but it should also be clear that mathematicians do not necessarily like that. Intuition can be misleading. Therefore, occasional unpleasant surprises and even catastrophic failures cannot be ruled out.

\section{B Sequence Transformations}

The fact that appropriate summation methods make it possible to use divergent series for computational purposes raises the question which of the numerous known summation techniques are best suited for the numerical evaluation of the divergent series occurring in this article. Typically, we are confronted with alternating series whose terms grow in magnitude like a fixed power of the index. Based on my own practical experience, I propose to use socalled sequence transformations, which are purely numerical techniques to transform a slowly convergent or divergent sequence $\left\{s_{n}\right\}_{n=0}^{\infty}$ to another sequence $\left\{s_{n}^{\prime}\right\}_{n=0}^{\infty}$ with hopefully better convergence properties.

For those interested in the history of sequence transformations, I recommend a monograph by Brezinski [21], which discusses earlier work starting from the 17 th century until 1945, as well as two articles by Brezinski [22, 23], which emphasize more recent developments.

The basic assumption of all sequence transformations is that the elements of a slowly convergent or divergent sequence $\left\{s_{n}\right\}_{n=0}^{\infty}$, which could be the partial sums $s_{n}=\sum_{k=0}^{n} a_{k}$ of an infinite series, can for all indices $n$ be partitioned into a (generalized) limit $s$ and a remainder 
or truncation error $r_{n}$ according to

$$
s_{n}=s+r_{n}, \quad n \in \mathbb{N}_{0} .
$$

If the sequence $\left\{s_{n}\right\}_{n=0}^{\infty}$ converges to $s$, the remainders $r_{n}$ in B.1 can be made negligible by increasing $n$ as much as necessary. But many sequences converge so slowly that this is not feasible. Increasing the index $n$ also does not help in the case of the divergent series.

Alternatively, one can try to improve convergence or accomplish a summation by computing approximations to the remainders $r_{n}$ which are then eliminated from the sequence elements $s_{n}$. At least conceptually, this is what a sequence transformation tries to do.

With the exception of a few practically more or less irrelevant model problems, sequence transformations can only eliminate approximations to the remainders. Thus, the elements of the transformed sequence $\left\{s_{n}^{\prime}\right\}_{n=0}^{\infty}$ are also be of the type of (B.1), which means that a transformed sequence element $s_{n}^{\prime}$ can also be partitioned into the same (generalized) limit $s$ and a transformed remainder $r_{n}^{\prime}$ according to

$$
s_{n}^{\prime}=s+r_{n}^{\prime}, \quad n \in \mathbb{N}_{0} .
$$

The transformed remainders $\left\{r_{n}^{\prime}\right\}_{n=0}^{\infty}$ are normally different from zero for all finite values of $n$. However, convergence is accelerated if the transformed remainders $\left\{r_{n}^{\prime}\right\}_{n=0}^{\infty}$ vanish more rapidly than the original remainders $\left\{r_{n}\right\}_{n=0}^{\infty}$, and a divergent sequence is summed if the transformed remainders vanish at all as $n \rightarrow \infty$.

Before the invention of electronic computers, mainly linear sequence transformations were used, which compute the elements of the transformed sequence $\left\{s_{n}^{\prime}\right\}_{n=0}^{\infty}$ as weighted averages of the elements of the input sequence $\left\{s_{n}\right\}_{n=0}^{\infty}$ according to

$$
s_{n}^{\prime}=\sum_{k=0}^{n} \mu_{n k} s_{k} .
$$

The theoretical properties of these matrix transformations are now very well understood and discussed in books by Hardy [78], Knopp [94], Petersen [105], Peyerimhoff [106], Zeller and Beekmann [166], Powell and Shah, and Boos [11]. Their main appeal lies in the fact that based on the work of Toeplitz [129] some necessary and sufficient conditions for the weights $\mu_{n k}$ in (B.3) could be formulated which guarantee that the application of such a matrix transformation to a convergent sequence $\left\{s_{n}\right\}_{n=0}^{\infty}$ yields a transformed sequence $\left\{s_{n}^{\prime}\right\}_{n=0}^{\infty}$ converging to the same limit $s=s_{\infty}$.

There are also some so-called Tauberian theorems which show rigorously that certain orders of for example Hölder's or Cesàro's summation method are needed to sum a divergent series whose terms grow in magnitude like a fixed power of the index [78, Theorem 39 on p. 95 and Theorem 49 on p. 103]. Since the terms of the divergent inner $\mu$ series considered in Sections 4 and 6 all grow in magnitude like a fixed power of the index, it looks like a natural idea to use either Hölder's or Cesàro's summation method for the summation of these divergent series. Unfortunately, the situation is not so simple. Since we also want to accomplish something useful if we only know a comparatively small number of numerically determined Laguerre series coefficients $\lambda_{n}^{(\alpha)}$, we should focus our attention on those purely numerical summation techniques that promise to be particularly efficient.

From a purely theoretical point of view, regularity is extremely desirable and greatly facilitates the formulation of nice mathematical proofs, but from a practical point of view, regularity is a serious disadvantage. This probably sounds paradoxical. However, Wimp remarks in the preface of his book [163, p. X] that the size of the domain of regularity of a transformation 
and its efficiency seem to be inversely related. Accordingly, regular matrix transformations are in general at most moderately powerful, and the popularity of most linear transformations as computational tools has declined considerably in recent years.

Nonlinear sequence transformations have largely complementary properties: They are nonregular, which means that the convergence of the transformed sequence is not guaranteed, let alone to the correct limit. In addition, their theoretical properties are far from being completely understood. Thus, from a purely theoretical point of view, nonlinear sequence transformations have many disadvantages, but they often accomplish spectacular transformation results which are clearly beyond the reach of regular matrix transformations. Consequently, nonlinear transformations now clearly dominate practical applications and - albeit to a lesser extend - also theoretical work. Detailed treatments of their theoretical properties and long lists of successful applications can be found in monographs by Brezinski [18, 19, 20], Brezinski and Redivo Zaglia [24], Cuyt [34, 35], Cuyt and Wuytack [37], Delahaye [40], Liem, Lü, and Shih [101], Marchuk and Shaidurov [103], Sidi [115], Walz [134], and Wimp [163], or in reviews by Caliceti, Meyer-Hermann, Ribeca, Surzhykov, and Jentschura [27], Homeier [81], and myself [136]).

In spite of their undeniable usefulness, nonlinear sequence transformations do not necessarily get the attention they deserve, in particular in the more theoretically oriented mathematical literature. For example, a (very) condensed review of the classical linear summability methods associated with the names of Cesàro, Abel, and Riesz can be found in Zayed's relatively recent book [165, Chapter 1.11.1], but the more powerful and computationally more useful nonlinear sequence transformations are not mentioned at all. Apparently, many mathematicians still prefer to work on the theoretically very nice, but computationally at most moderately powerful regular matrix transformations.

Nevertheless, there are encouraging signs that the situation is changing for the better, and there are now several books by mathematicians that describe how nonlinear sequence transformations can be employed effectively as computational tools. For example, the most recent (third) edition of the book Numerical Recipes [108] now also discusses nonlinear sequence transformations (for a discussion of the topics treated there and for further details, see [153]).

I can also recommend a recent book by Bornemann, Laurie, Wagon, and Waldvogel [16] on extreme digit hunting in the context of some challenging problems of numerical analysis. For this extreme digit hunting, the authors also use sequence transformations, whose basic theory is described compactly in their Appendix A. This Appendix is too short to provide a reasonably complete and balanced presentation of sequence transformation, but I think that a novice can benefit considerably from reading it. I also like the extremely pragmatic approach of the authors of this book, which is very uncommon among mathematicians. Probably, this is due to the fact that the authors are not primarily interested in the mathematical theory of sequence transformations: They only wanted to apply sequence transformations as computational tools in order to obtain more precise results at tolerable computational costs.

Then, there is a very recent book by Gil, Segura, and Temme [48] on the evaluation of special functions. It discusses in addition to various other computational techniques also Padé approximants, continued fractions, and nonlinear sequence transformations which all facilitate the evaluation of (power) series representations for special functions.

My own research shows that nonlinear sequence transformations can be extremely useful in a large variety of different contexts. I applied them successfully in such diverse fields as the evaluation of molecular multicenter integrals of exponentially decaying functions [52, 82, 122, 158, 161], the evaluation of special functions and related objects [84, 85, 136, 137, 139, 140, $143,147,148,155,162$, the summation of strongly divergent quantum mechanical perturbation expansions [8, 31, 32, 33, 83, 86, 137, 138, 140, 141, 142, 144, 145, 147, 156, 157], the 
prediction of unknown perturbation series coefficients [8, 83, 86, 145, [146], and the extrapolation of quantum chemical crystal orbital and cluster electronic structure calculations for oligomers to their infinite chain limits of stereoregular quasi-onedimensional organic polymers [30, 159, 160].

In view of all these examples, it is probably justified to claim that anybody involved in computational work should have at least some basic knowledge about the power and also about the shortcomings and limitations of nonlinear sequence transformations.

Padé approximants $[m / n]_{f}(z)$ can be viewed to be a special class of nonlinear sequence transformation since they convert the partial sums $f_{n}(z)=\sum_{k=0}^{n} \gamma_{k} z^{k}$ of a (formal) power series for some function $f(z)$ to a doubly indexed sequence of rational functions. As documented by the long list of successful applications in the monograph by Baker and Graves-Morris [3], Padé approximants are now almost routinely used in theoretical physics and in applied mathematics to overcome problems with slowly convergent or divergent power series. It is, however, not nearly so well known among nonspecialists that alternative sequence transformations can at least for certain computational problems be much more effective than Padé approximants.

The probably best known sequence transformation is Wynn's epsilon algorithm [164], which is defined by the following nonlinear recursive scheme:

$$
\begin{aligned}
& \varepsilon_{-1}^{(n)}=0, \quad \varepsilon_{0}^{(n)}=s_{n}, \quad n \in \mathbb{N}_{0}, \\
& \varepsilon_{k+1}^{(n)}=\varepsilon_{k-1}^{(n+1)}+\frac{1}{\varepsilon_{k}^{(n+1)}-\varepsilon_{k}^{(n)}}, \quad k, n \in \mathbb{N}_{0} .
\end{aligned}
$$

The elements $\varepsilon_{2 k}^{(n)}$ with even subscripts provide approximations to the (generalized) limit $s$ of the sequence $\left\{s_{n}\right\}_{n=0}^{\infty}$ to be transformed, whereas the elements $\varepsilon_{2 k+1}^{(n)}$ with odd subscripts are only auxiliary quantities which diverge if the whole process converges. A compact FORTRAN 77 program for the epsilon algorithm as well as the underlying computational algorithm is described in [136, Section 4.3]. In [108, p. 213], a translation of this FORTRAN 77 program to $\mathrm{C}$ can be found.

If the elements of the input sequence $\left\{s_{n}\right\}_{n=0}^{\infty}$ are the partial sums $f_{n}(z)=\sum_{k=0}^{n} \gamma_{k} z^{k}$ of the (formal) power series for some function $f(z)$, then the epsilon algorithm with $\varepsilon_{0}^{(n)}=f_{n}(z)$ produces Padé approximants to $f(z)$ :

$$
\varepsilon_{2 k}^{(n)}=[n+k / k]_{f}(z), \quad k, n \in \mathbb{N}_{0}
$$

But Wynn's epsilon algorithm is not restricted to input data that are the partial sums of a (formal) power series. Therefore, it is more general and more widely applicable than Padé approximants. Moreover, the epsilon algorithm can be generalized to cover for example vector sequences. A recent review can be found in [51].

Since the epsilon algorithm can be used for the computation of Padé approximants, it is discussed in books on Padé approximants such as the one by Baker and Graves-Morris [3], but there is also an extensive literature dealing directly with it. On p. 120 of Wimps book [163] it is mentioned that over 50 articles on the epsilon algorithm were published by Wynn alone, and at least 30 articles by Brezinski. As a fairly complete source of references on the epsilon algorithm, Wimp recommends Brezinski's first book [18]. However, this book was published in 1977, and since then many more articles on the theory or on applications of Wynn's epsilon algorithm have appeared. Thus, any attempt of providing a reasonably complete bibliography would be beyond the scope of this article.

In a convergence acceleration or summation process, it is usually a good idea to try to use the available information as effectively as possible (possible exceptions to this rule are 
discussed in [147]). Let us assume that a finite subset $\left\{s_{0}, s_{1}, \ldots, s_{m}\right\}$ of sequence elements is available. Then, those elements $\varepsilon_{2 k}^{(n)}$ of the epsilon table produced by (B.4) should be chosen as approximations to the limit of the imput sequence that have the highest possible subscript or transformation order. Thus, if $m \in \mathbb{N}_{0}$ is even, $m=2 \mu$, I use as approximation to the limit of the input sequence the transformation [136, Eq. (4.3-4)]

$$
\left\{s_{0}, s_{1}, \ldots, s_{2 \mu}\right\} \rightarrow \varepsilon_{2 \mu}^{(0)},
$$

and if $m \in \mathbb{N}_{0}$ is odd, $m=2 \mu+1$, I use the transformation [136. Eq. (4.3-5)]

$$
\left\{s_{1}, s_{2}, \ldots, s_{2 \mu+1}\right\} \rightarrow \varepsilon_{2 \mu}^{(1)} .
$$

With the help of the notation $\lfloor x\rfloor$ for the integral part of $x$, which is the largest integer $v$ satisfying $v \leq x$, these two relationships can be combined into a single equation yielding [136, Eq. (4.3-6)]:

$$
\left\{s_{m-2\lfloor m / 2\rfloor}, s_{m-2\lfloor m / 2\rfloor+1}, \ldots, s_{m}\right\} \rightarrow \varepsilon_{2\lfloor m / 2\rfloor}^{(m-2\lfloor m / 2\rfloor)} .
$$

Wynn's epsilon algorithm is an example of a sequence transformation that uses as input data only the elements of the sequence to be transformed. However, in some cases structural information on the dependence of the sequence elements $s_{n}$ on the index $n$ is available. For example, it is well known that the truncation error of a convergent series with strictly alternating and monotonously decreasing terms is bounded in magnitude by the first term not included in the partial sum and that it possesses the same sign as this term (see for instance [94, p. 259]). The first term neglected is also the best simple estimate for the truncation error of a strictly alternating nonterminating and thus diverging hypergeometric series ${ }_{2} F_{0}(\alpha, \beta ;-z)$ with $\alpha, \beta, z>0$ [29, Theorem 5.12-5]. Such an information on the index dependence of the truncation errors should be extremely helpful in a convergence acceleration or summation process, but a sequence transformations like Wynn's epsilon algorithm cannot benefit from it.

A convenient way of incorporating such an information into the transformation process consists in the use of remainder estimates $\left\{\omega_{n}\right\}_{n=0}^{\infty}$. Because of the additional information contained in the remainder estimates, sequence transformations of that kind are potentially very powerful as well as very versatile.

The best-known example of such a sequence transformation is Levin's transformation [100], which is generally considered to be a very powerful as well as very versatile sequence transformation (see for example [24, 81, 120, 121, 136, 149] and references therein):

$$
\mathscr{L}_{k}^{(n)}\left(\beta, s_{n}, \omega_{n}\right)=\frac{\sum_{j=0}^{k}(-1)^{j}\left(\begin{array}{l}
k \\
j
\end{array}\right) \frac{(\beta+n+j)^{k-1}}{(\beta+n+k)^{k-1}} \frac{s_{n+j}}{\omega_{n+j}}}{\sum_{j=0}^{k}(-1)^{j}\left(\begin{array}{l}
k \\
j
\end{array}\right) \frac{(\beta+n+j)^{k-1}}{(\beta+n+k)^{k-1}} \frac{1}{\omega_{n+j}}}, \quad k, n \in \mathbb{N}_{0} .
$$

Here, $\beta>0$ is a shift parameter. The most obvious choice is $\beta=1$, which is exclusively used in this article.

The numerator and denominator sums of $\mathscr{L}_{k}^{(n)}\left(\beta, s_{n}, \omega_{n}\right)$ can also be computed recursively ([136, Eq. (7.2-8) - (7.2-10)] or in [149, Eq. (3.11)]):

$$
\begin{aligned}
L_{0}^{(n)} & =u_{n}, \quad n \in \mathbb{N}_{0}, \\
L_{k+1}^{(n)} & =L_{k}^{(n+1)}-\frac{(\beta+n)(\beta+n+k)^{k-1}}{(\beta+n+k+1)^{k}} L_{k}^{(n)}, \quad k, n \in \mathbb{N}_{0},
\end{aligned}
$$


By choosing in B.10a either $u_{n}=s_{n} / \omega_{n}$ or $u_{n}=1 / \omega_{n}$, we obtain the numerator and denominator sums of Levin's transformation (B.9).

As discussed in more detail in [136, 149], Levin's transformation is based on the implicit assumption that the ratio $\left[s_{n}-s\right] / \omega_{n}$ can be expressed as a power series in $1 /(n+\beta)$. A different class of sequence transformations can be derived by assuming that $\left[s_{n}-s\right] / \omega_{n}$ can be expressed as a so-called factorial series, yielding [136, Eq. (8.2-7)]

$$
\mathscr{S}_{k}^{(n)}\left(\beta, s_{n}, \omega_{n}\right)=\frac{\sum_{j=0}^{k}(-1)^{j}\left(\begin{array}{l}
k \\
j
\end{array}\right) \frac{(\beta+n+j)_{k-1}}{(\beta+n+k)_{k-1}} \frac{s_{n+j}}{\omega_{n+j}}}{\sum_{j=0}^{k}(-1)^{j}\left(\begin{array}{l}
k \\
j
\end{array}\right) \frac{(\beta+n+j)_{k-1}}{(\beta+n+k)_{k-1}} \frac{1}{\omega_{n+j}}}, \quad k, n \in \mathbb{N}_{0} .
$$

As in the case of Levin's transformation. $\beta>0$ is a shift parameter, and again, only $\beta=1$ is considered in this article. Formally, we obtain $\mathscr{S}_{k}^{(n)}\left(\beta, s_{n}, \omega_{n}\right)$ from $\mathscr{L}_{k}^{(n)}\left(\beta, s_{n}, \omega_{n}\right)$ if we replace in (B.9) the powers $(\beta+n+j)^{k-1}$ by Pochhammer symbols $(\beta+n+j)_{k-1}$.

The numerator and denominator sums of $\mathscr{S}_{k}^{(n)}\left(\beta, s_{n}, \omega_{n}\right)$ can also be computed recursively ([136, Eq. (7.2-8) - (8.3-7)] or in [149, Eq. (3.12)]):

$$
\begin{aligned}
S_{0}^{(n)} & =u_{n}, \quad n \in \mathbb{N}_{0}, \\
S_{k+1}^{(n)} & =S_{k}^{(n+1)}-\frac{(\beta+n+k-1)(\beta+n+k)}{(\beta+n+2 k-1)(\beta+n+2 k)} S_{k}^{(n)}, \quad k, n \in \mathbb{N}_{0},
\end{aligned}
$$

The initial values $u_{n}=s_{n} / \omega_{n}$ produce the numerators of the transformation (B.11), and the initial values $u_{n}=1 / \omega_{n}$ yield the denominators.

Both Levin's sequence transformation (B.9) as well as the related transformation B.11 utilize the information contained in explicit remainder estimates $\left\{\omega_{n}\right\}_{n=0}^{\infty}$ which should be chosen in such a way that the ratio $\left[s_{n}-s\right] / \omega_{n}$ becomes a smooth function of $n$ that can be annihilated effectively by weighted finite difference operators (see for example [149, Sections II and IV] and references therein). Accordingly, the choice of the remainder estimates is of utmost importance for the success or failure of a convergence acceleration or summation process involving Levin-type transformations. In this respect, it may be interesting to note that a symbolic approach for the construction of asymptotic estimates to the truncation errors of series representations for special functions was recently developed in [150].

On the basis of purely heuristic arguments Levin [100] had suggested some simple remainder estimates which according to experience work remarkably well in a large variety of cases and which can also be used in the case of the sequence transformation (B.11). But the best simple estimate for the truncation error of a strictly alternating convergent series is the first term not included in the partial sum [94, p. 259]. Moreover, the first term neglected is also an estimate of the truncation error of a divergent hypergeometric series ${ }_{2} F_{0}(a, b,-z)$ with $a, b, z>0$ [29, Theorem 5.12-5]. Accordingly, Smith and Ford [120] proposed for sequences of partial sums of alternating series the following remainder estimate:

$$
\omega_{n}=\Delta s_{n} .
$$

The use of this remainder estimate in [100] and (B.11) yields the following variants of the sequence transformations $\mathscr{L}_{k}^{(n)}\left(\beta, s_{n}, \omega_{n}\right)$ and $\mathscr{S}_{k}^{(n)}\left(\beta, s_{n}, \omega_{n}\right)$ :

$$
\begin{aligned}
& d_{k}^{(n)}\left(\beta, s_{n}\right)=\mathscr{L}_{k}^{(n)}\left(\beta, s_{n}, \Delta s_{n}\right), \\
& \delta_{k}^{(n)}\left(\beta, s_{n}\right)=\mathscr{S}_{k}^{(n)}\left(\beta, s_{n}, \Delta s_{n}\right) .
\end{aligned}
$$


If the elements of the input sequence $\left\{s_{n}\right\}_{n=0}^{\infty}$ are the partial sums $f_{n}(z)=\sum_{k=0}^{n} \gamma_{k} z^{k}$ of the (formal) power series for some function $f(z)$, then $d_{k}^{(n)}\left(\beta, f_{n}(z)\right)$ and $\delta_{k}^{(n)}\left(\beta, f_{n}(z)\right)$ are ratios of two polynomials in $z$ of degrees $k+n$ and $k$, respectively (for a detailed discussion of these rational approximants, see [149, Section VI]).

The transformations $d_{k}^{(n)}\left(\beta, s_{n}\right)$ and in particular also $\delta_{k}^{(n)}\left(\beta, s_{n}\right)$ were found to be remarkably powerful summation techniques for divergent series with strictly alternating terms. Numerous successful applications of Levin's sequence transformation (B.9) and of the related transformation $(B .11$ and their variants in convergence acceleration and summation processes are described in [149, pp. 1210 and 1225]. It may be interesting to note that recently $\delta_{k}^{(n)}\left(\beta, s_{n}\right)$ has been used quite a lot in optics [12, 13, 14, 15].

In the case of the transformations $(\mathbb{B . 1 4})$ and $(\mathbb{B . 1 5})$, the approximation to the limit with the highest transformation order is given by

$$
\left\{s_{0}, s_{1}, \ldots, s_{m+1}\right\} \rightarrow \Xi_{m}^{(0)}\left(\beta, s_{0}\right),
$$

where $\Xi_{k}^{(n)}\left(\beta, s_{n}\right)$ stands for either $d_{k}^{(n)}\left(\beta, s_{n}\right)$ or $\delta_{k}^{(n)}\left(\beta, s_{n}\right)$.

\section{References}

[1] Abramowitz, M. and Stegun, I. A. (editors), Handbook of Mathematical Functions (National Bureau of Standards, Washington, D. C., 1972).

[2] Askey, R. and Wainger, S., Mean convergecne of expansions in Laguerre and Hermite series, Amer. J. Math. 87, 695 - 708 (1965).

[3] Baker, Jr., G. A. and Graves-Morris, P., Padé Approximants (Cambridge U. P., Cambridge, 1996), 2nd edition.

[4] Balser, W., From Divergent Power Series to Analytic Functions (Springer-Verlag, Berlin, 1994).

[5] Balser, W., Formal Power Series and Linear Systems of Meromorphic Ordinary Differential Equations (Springer-Verlag, New York, 1999).

[6] Barbeau, E. J., Euler subdues a very obstreperous series, Amer. Math. Monthly 86, 356 - 372 (1979).

[7] Barbeau, E. J. and Leah, P. J., Euler's 1760 paper on divergent series, Hist. Math. 3, $141-160$ (1976).

[8] Bender, C. M. and Weniger, E. J., Numerical evidence that the perturbation expansion for a non-Hermitian $\mathscr{P} \mathscr{T}$-symmetric Hamiltonian is Stieltjes, J. Math. Phys. 42, 2167 -2183 (2001).

[9] Bender, C. M. and Wu, T. T., Anharmonic oscillator, Phys. Rev. 184, 1231 - 1260 (1969).

[10] Bender, C. M. and Wu, T. T., Large-order behavior of perturbation theory, Phys. Rev. Lett. 27, $461-465$ (1971).

[11] Boos, J., Classical and Modern Methods of Summability (Oxford U. P., Oxford, 2000). 
[12] Borghi, R., Evaluation of diffraction catastrophes by using Weniger transformation, Opt. Lett. 32, 226 - 228 (2007).

[13] Borghi, R., Summing Pauli asymptotic series to solve the wedge problem, J. Opt. Soc. Amer. A 25, 211 - 218 (2008).

[14] Borghi, R. and Alonso, M., Efficient evaluation of far-field asymptotic series, in ICTON '07: 9th International Conference on Transparent Optical Networks, volume 3, 246 250 (IEEE Xplore, Piscataway, NJ, 2007).

[15] Borghi, R. and Santarsiero, M., Summing Lax series for nonparaxial beam propagation, Opt. Lett. 28, $774-776$ (2003).

[16] Bornemann, F., Laurie, D., Wagon, S., and Waldvogel, J., The SIAM 100-Digit Challenge: A Study in High-Accuracy Numerical Computing (Society of Industrial Applied Mathematics, Philadelphia, 2004).

[17] Boutet de Monvel, L. (editor), Méthodes Résurgentes (Hermann, Paris, 1994).

[18] Brezinski, C., Accélération de la Convergence en Analyse Numérique (Springer-Verlag, Berlin, 1977).

[19] Brezinski, C., Algorithmes d'Accélération de la Convergence - Étude Numérique (Éditions Technip, Paris, 1978).

[20] Brezinski, C., Padé-Type Approximation and General Orthogonal Polynomials (Birkhäuser, Basel, 1980).

[21] Brezinski, C., History of Continued Fractions and Padé Approximants (SpringerVerlag, Berlin, 1991).

[22] Brezinski, C., Extrapolation algorithms and Padé approximations: A historical survey, Appl. Numer. Math. 20, 299 - 318 (1996).

[23] Brezinski, C., Convergence acceleration during the 20th century, J. Comput. Appl. Math. 122, 1 - 21 (2000). Reprinted in Brezinski, C. (editor), Numerical Analysis 2000, Vol. 2: Interpolation and Extrapolation, 1 - 21 (Elsevier, Amsterdam, 2000).

[24] Brezinski, C. and Redivo Zaglia, M., Extrapolation Methods (North-Holland, Amsterdam, 1991).

[25] Bühring, W., An analytic continuation formula for the generalized hypergeometric function, SIAM J. Math. Anal. 19, 1249 - 1251 (1988).

[26] Burkhardt, H., Über den Gebrauch divergenter Reihen in der Zeit von 1750 - 1860, Math. Annal. 70, 169 - 206 (1911).

[27] Caliceti, E., Meyer-Hermann, M., Ribeca, P., Surzhykov, A., and Jentschura, U. D., From useful algorithms for slowly convergent series to physical predictions based on divergent perturbative expansions, Phys. Rep. 446, 1 - 96 (2007).

[28] Candelpergher, B., Nosmas, J. C., and Pham, F., Approche de la Résurgence (Hermann, Paris, 1993). 
[29] Carlson, B. C., Special Functions of Applied Mathematics (Academic Press, New York, 1977).

[30] Cioslowski, J. and Weniger, E. J., Bulk properties from finite cluster calculations. VIII. Benchmark calculations on the efficiency of extrapolation methods for the HF and MP2 energies of polyacenes, J. Comput. Chem. 14, 1468 - 1481 (1993).

[31] Č́ižek, J., Vinette, F., and Weniger, E. J., Examples on the use of symbolic computation in physics and chemistry: Applications of the inner projection technique and of a new summation method for divergent series, Int. J. Quantum Chem. Symp. 25, $209-223$ (1991).

[32] Č́ižek, J., Vinette, F., and Weniger, E. J., On the use of the symbolic language Maple in physics and chemistry: Several examples, in de Groot, R. A. and Nadrchal, J. (editors), Proceedings of the Fourth International Conference on Computational Physics PHYSICS COMPUTING '92, 31 - 44 (World Scientific, Singapore, 1993). Reprinted as J. Mod. Phys. C 4, $257-270$ (1993).

[33] Č́ižek, J., Weniger, E. J., Bracken, P., and Špirko, V., Effective characteristic polynomials and two-point Padé approximants as summation techniques for the strongly divergent perturbation expansions of the ground state energies of anharmonic oscillators, Phys. Rev. E 53, 2925 - 2939 (1996).

[34] Cuyt, A. (editor), Nonlinear Numerical Methods and Rational Approximation (Reidel, Dordrecht, 1988).

[35] Cuyt, A. (editor), Nonlinear Numerical Methods and Rational Approximation II (Kluwer, Dordrecht, 1994).

[36] Cuyt, A., Brevik Petersen, V., Verdonk, B., Waadeland, H., and Jones, W. B., Handbook of Continued Fractions for Special Functions (Springer-Verlag, New York, 2008).

[37] Cuyt, A. and Wuytack, L., Nonlinear Methods in Numerical Analysis (North-Holland, Amsterdam, 1987).

[38] Davis, H. F., Fourier series and Orthogonal Functions (Dover, New York, 1989). Originally published by Allyn and Bacon (Boston, 1963).

[39] Delabaere, E. and Pham, F., Resurgent methods in semi-classical asymptotics, Ann. Inst. Henri Poincaré Phys. Théor. 71, 1 - 94 (1999).

[40] Delahaye, J.-P., Sequence Transformations (Springer-Verlag, Berlin, 1988).

[41] Doha, E. H., On the connection coefficients and recurrence relations arising from expansions in series of Laguerre polynomials, J. Phys. A 36, 5449 - 5462 (2003).

[42] Dyson, D. J., Divergence of perturbation theory in quantum electrodynamics, Phys. Rev. 85, 32 - 33 (1952).

[43] Erdélyi, A., Magnus, W., Oberhettinger, F., and Tricomi, F. G., Higher Transcendental Functions, volume II (McGraw-Hill, New York, 1953).

[44] Ferraro, G., The Rise and Development of the Theory of Series up to the Early 1820s (Springer-Verlag, New York, 2008). 
[45] Filter, E. and Steinborn, E. O., A matrix representation of the translation operator with respect to a basis of exponentially declining functions, J. Math. Phys. 21, $2725-2736$ (1980).

[46] Ford, W. F. and Pennline, J. A., When does convergence in the mean imply uniform convergence?, Amer. Math. Monthly 114, 58 - 60 (2007).

[47] Frobenius, G., Ueber die Leibnitzsche Reihe, J. reine angew. Math. 89, 262 - 264 (1880).

[48] Gil, A., Segura, J., and Temme, N. M., Numerical Methods for Special Functions (SIAM, Philadelphia, 2007).

[49] Gottlieb, D. and Orszag, S. A., Numerical Analysis of Spectral Methods: Theory and Applications, Regional Conference Series in Applied Mathematics (SIAM, Philadelphia, 1977).

[50] Gradshteyn, I. S. and Rhyzhik, I. M., Table of Integrals, Series, and Products (Academic Press, Boston, 1994), 5th edition.

[51] Graves-Morris, P. R., Roberts, D. E., and Salam, A., The epsilon algorithm and related topics, J. Comput. Appl. Math. 122, 51 - 80 (2000). Reprinted in Brezinski, C. (editor), Numerical Analysis 2000, Vol. 2: Interpolation and Extrapolation, 51 - 80 (Elsevier, Amsterdam, 2000).

[52] Grotendorst, J., Weniger, E. J., and Steinborn, E. O., Efficient evaluation of infiniteseries representations for overlap, two-center nuclear attraction, and Coulomb integrals using nonlinear convergence accelerators, Phys. Rev. A 33, 3706 - 3726 (1986).

[53] Guseinov, I. I., Expansion of Slater-type orbitals about a new origin and analytical evaluation of multicenter electron-repulsion integrals, Phy. Rev. A 22, 369 - 371 (1980).

[54] Guseinov, I. I., Evaluation of expansion coefficients for translation of Slater-type orbitals using complete orthonormal sets of exponential-type functions, Int. J. Quantum Chem. 81, 126 - 129 (2001).

[55] Guseinov, I. I., Computation of molecular integrals over Slater-type orbitals. IX. Calculation of multicenter multielectron molecular integrals with integer and noninteger $n$ Slater orbitals using complete orthonormal sets of exponential functions, J. Mol. Struc. (Theochem) 593, $65-69$ (2002).

[56] Guseinov, I. I., New complete orthonormal sets of exponential-type orbitals and their application to translation of Slater orbitals, Int. J. Quantum Chem. 90, $114-118$ (2002).

[57] Guseinov, I. I., Unified analytical treatment of one-electron multicenter integrals of central and noncentral potentials over Slater orbitals, Int. J. Quantum Chem. 90, 980 985 (2002).

[58] Guseinov, I. I., Unified treatment of integer and noninteger $n$ multicenter multielectron molecular integrals using complete orthonormal sets of $\Psi^{\alpha}$-ETOs, J. Mol. Struc. (Theochem) 625, 221 - 225 (2003). 
[59] Guseinov, I. I., Addition theorems for Slater-type orbitals and their application to multicenter multielectron integrals of central and noncentral interaction potentials, J. Mol. Model. 9, 190 - 194 (2003).

[60] Guseinov, I. I., Unified analytical treatment of multicenter multielectron integrals of central and noncentral interaction potentials over Slater orbitals using $\Psi^{\alpha}$-ETOs, $J$. Chem. Phys. 119, 4614 - 4619 (2003).

[61] Guseinov, I. I., Unified analytical treatment of multicentre electron attraction, electric field and electric field gradient integrals over Slater orbitals, J. Phys. A 37, $957-964$ (2004).

[62] Guseinov, I. I., Use of $\Psi^{\alpha}$-ETOs in the unified treatment of electronic attraction, electric field and electric field gradient multicenter integrals of screened Coulomb potentials over Slater orbitals, J. Mol. Model. 10, 19 - 24 (2004). Erratum, J. Mol. Model. 10, 233 (2004).

[63] Guseinov, I. I., Unified treatment of multicenter integrals of integer and noninteger $u$ Yukawa-type screened Coulomb type potentials and their derivatives over Slater orbitals, J. Chem. Phys. 120, 9454 - 9457 (2004).

[64] Guseinov, I. I., One-range addition theorems for derivatives of Slater-type orbitals, $J$. Mol. Model. 10, 212 - 215 (2004).

[65] Guseinov, I. I., Unified analytical treatment of two-electron multicenter integrals of central and noncentral interaction potentials over Slater orbitals, Int. J. Quantum Chem. 100, 206 - 207 (2004).

[66] Guseinov, I. I., Unified treatment of electronic attraction, electric field, and electricfield gradient multicenter integrals of screened and nonscreened Coulomb potentials using overlap integrals for Slater orbitals, Can. J. Phys. 82, 819 - 825 (2004).

[67] Guseinov, I. I., One-range addition theorems for Coulomb interaction potential and its derivatives, Chem. Phys. 309, $209-213$ (2005).

[68] Guseinov, I. I., One-range addition theorems for Yukawa-like central and noncentral interaction potentials and their derivatives, Bull. Chem. Soc. Japan 78, 611 - 614 (2005).

[69] Guseinov, I. I., One-range addition theorems for derivatives of integer and noninteger $u$ Coulomb Yukawa type central and noncentral potentials and their application to multicenter integrals of integer and noninteger $n$ Slater orbitals, J. Mol. Struc. (Theochem) 757, 165 - 169 (2005).

[70] Guseinov, I. I., One-range addition theorems for combined Coulomb and Yukawa like central and noncentral interaction potentials and their derivatives, J. Math. Chem. 39, $253-258$ (2006).

[71] Guseinov, I. I., Combined Extended Rejoinder to "Extended Comment on "One-Range Addition Theorems for Coulomb Interaction Potential and Its Derivatives" by I. I. Guseinov (Chem. Phys. and Vol. 309 (2005)”, pp. 209-213), Los Alamos Preprint arXiv:0706.0975v3 [physics.chem-ph] (http://arXiv.org) (2007). 
[72] Guseinov, I. I. and Mamedov, B. A., Use of addition theorems in evaluation of multicenter nuclear-attraction and electron-repulsion integrals with integer and noninteger $n$ Slater-type orbitals, Theor. Chem. Acc. 108, 21 - 26 (2002).

[73] Guseinov, I. I. and Mamedov, B. A., Unified treatment of overlap integrals with integer and noninteger $n$ Slater-type orbitals using translational and rotational transformations for spherical harmonics, Can. J. Phys. 82, 205 - 211 (2004).

[74] Guseinov, I. I. and Mamedov, B. A., Evaluation of multicenter one-electron integrals of noninteger $u$ screened Coulomb type potentials and their derivatives over noninteger $n$ Slater orbitals, J. Chem. Phys. 121, 1649 - 1654 (2004).

[75] Guseinov, I. I. and Mamedov, B. A., Evaluation of multicenter electronic attraction, electric field and electric field gradient integrals with screened and nonscreened Coulomb potentials over integer and noninteger $n$ Slater orbitals, J. Math. Chem. 36, 113 - 121 (2004).

[76] Guseinov, I. I. and Mamedov, B. A., Calculation of multicenter electronic attraction, electric field and electric field gradient integrals of Coulomb potential over integer and noninteger $n$ Slater orbitals, J. Math. Chem. 37, 353 - 364 (2005).

[77] Guseinov, I. I., Mamedov, B. A., and Sünel, N., Computation of molecular integrals over Slater-type orbitals. X. Calculation of overlap integrals with integer and noninteger $n$ Slater orbitals using complete orthonormal sets of exponential functions, J. Mol. Struc. (Theochem) 593, 71 - 77 (2002).

[78] Hardy, G. H., Divergent Series (Clarendon Press, Oxford, 1949).

[79] Higgins, J. R., Completeness and Basis Properties of Sets of Special Functions (Cambridge U. P., Cambridge, 1977).

[80] Hölder, O., Grenzwerthe von Reihen an der Konvergenzgrenze, Math. Annal. 20, 535 -549 (1882).

[81] Homeier, H. H. H., Scalar Levin-type sequence transformations, J. Comput. Appl. Math. 122, 81 - 147 (2000). Reprinted in Brezinski, C. (editor), Numerical Analysis 2000, Vol. 2: Interpolation and Extrapolation, 81 - 147 (Elsevier, Amsterdam, 2000).

[82] Homeier, H. H. H. and Weniger, E. J., On remainder estimates for Levin-type sequence transformations, Comput. Phys. Commun. 92, 1 - 10 (1995).

[83] Jentschura, U. D., Becher, J., Weniger, E. J., and Soff, G., Resummation of QED perturbation series by sequence transformations and the prediction of perturbative coefficients, Phys. Rev. Lett. 85, 2446 - 2449 (2000).

[84] Jentschura, U. D., Gies, H., Valluri, S. R., Lamm, D., and Weniger, E., QED effective action revisited, Can. J. Phys. 80, 267 - 284 (2002).

[85] Jentschura, U. D., Mohr, P. J., Soff, G., and Weniger, E. J., Convergence acceleration via combined nonlinear-condensation transformations, Comput. Phys. Commun. 116, 28 - 54 (1999).

[86] Jentschura, U. D., Weniger, E. J., and Soff, G., Asymptotic improvement of resummations and perturbative predictions in quantum field theory, J. Phys. G 26, $1545-1568$ (2000). 
[87] Kaijser, P. and Smith, Jr., V. H., Evaluation of momentum distributions and Compton profiles for atomic and molecular systems, Adv. Quantum Chem. 10, 37 - 76 (1977).

[88] Klahn, B., Die Konvergenz des Ritz'schen Variationsverfahrens in der Quantenchemie, Ph.D. thesis, Mathematisch-Naturwissenschaftliche Fakultät der Georg-August-Universität zu Göttingen, Göttingen (1975).

[89] Klahn, B., Review of linear independence properties of infinite sets of functions used in quantum chemistry, Adv. Quantum Chem. 13, 155 - 209 (1981).

[90] Klahn, B. and Bingel, W. A., The convergence of the Rayleigh-Ritz method in quantum chemistry. I. the criteria for convergence, Theor. Chim. Acta 44, 9 - 26 (1977).

[91] Klahn, B. and Bingel, W. A., The convergence of the Rayleigh-Ritz method in quantum chemistry. II. investigation of the convergence for special systems of Slater, Gauss and two-electron functions, Theor. Chim. Acta 44, 27 - 43 (1977).

[92] Klahn, B. and Bingel, W. A., Completeness and linear independence of basis sets used in quantum chemistry, Int. J. Quantum Chem. 11, 943 - 957 (1977).

[93] Klahn, B. and Morgan III, J. D., Rates of convergence of variational calculations and of expectation values, J. Chem. Phys. 81, $410-433$ (1984).

[94] Knopp, K., Theorie und Anwendung der unendlichen Reihen (Springer-Verlag, Berlin, 1964).

[95] Kochneff, E., Expansions in Laguerre polynomials of negative order, J. Approx. Theory 81, 332 - 346 (1995).

[96] Körner, T. W., Fourier Analysis (Cambridge U. P., Cambridge, 1988).

[97] Kozlov, V. V., Euler and mathematical methods in mechanics (on the 300th anniversary of the birth of Leonhard Euler), Russ. Math. Surv. 62, 639 - 661 (2007).

[98] Kranz, H. H. and Steinborn, E. O., Implications and improvements of single-center expansions in molecules, Phys. Rev. A 25, 66 - 75 (1982).

[99] Le Guillou, J. C. and Zinn-Justin, J. (editors), Large-Order Behaviour of Perturbation Theory (North-Holland, Amsterdam, 1990).

[100] Levin, D., Development of non-linear transformations for improving convergence of sequences, Int. J. Comput. Math. B 3, 371 - 388 (1973).

[101] Liem, C. B., Lü, T., and Shih, T. M., The Splitting Extrapolation Method (World Scientific, Singapore, 1995).

[102] Magnus, W., Oberhettinger, F., and Soni, R. P., Formulas and Theorems for the Special Functions of Mathematical Physics (Springer-Verlag, New York, 1966).

[103] Marchuk, G. I. and Shaidurov, V. V., Difference Methods and Their Extrapolations (Springer-Verlag, New York, 1983).

[104] Olver, F. W. J., Asymptotics and Special Functions (A. K. Peters, Natick, Mass., 1997). Originally published by Academic Press (New York, 1974). 
[105] Petersen, G. M., Regular Matrix Transformations (McGraw-Hill, London, 1966).

[106] Peyerimhoff, A., Lectures on Summability (Springer-Verlag, Berlin, 1969).

[107] Pollard, H., Representation of an analytic function by a Laguerre series, Ann. Math. 48, 358 - 365 (1947).

[108] Press, W. H., Teukolsky, S. A., Vetterling, W. T., and Flannery, B. P., Numerical Recipes: The Art of Scientific Computing (Cambridge U. P., Cambridge, 2007), 3rd edition.

[109] Rainville, E. D., Special Functions (Chelsea, Bronx, New York, 1971). Originally published by Macmillan (New York, 1960).

[110] Rubel, L. A., The editor's corner: Summability theory: A neglected tool of analysis, Amer. Math. Monthly 96, 421 - 423 (1989).

[111] Rusev, P., Expansion of analytic functions in series of classical orthogonal polynomials, Banach Cent. Publ. 11, 287 - 298 (1983).

[112] S., V. V., Euler and his work on infinite series, Bull. Amer. Math. Soc. 44, 515 - 539 (2007).

[113] Sánchez-Ruiz, J., López-Artéz, P., and Dehesa, J. S., Expansions in series of varying Laguerre polynomials and some applications to molecular potentials, J. Comput. Appl. Math. 153, 411 - 421 (2003).

[114] Sansone, G., Orthogonal Functions (Krieger, Huntington, NY, 1977). Revised edition. Originally published by Wiley (New York, 1959).

[115] Sidi, A., Practical Extrapolation Methods (Cambridge U. P., Cambridge, 2003).

[116] Skorokhodov, S. L., Advanced techniques for computing divergent series, Nucl. Inst. Meth. Phys. Res. A 502, 636 - 638 (2003).

[117] Slater, J. C., Atomic shielding constants, Phys. Rev. 36, 57 - 64 (1930).

[118] Slater, J. C., Analytic atomic wave functions, Phys. Rev. 42, 33 - 43 (1932).

[119] Slater, L., Confluent Hypergeometric Functions (Cambridge U. P., Cambridge, 1960).

[120] Smith, D. A. and Ford, W. F., Acceleration of linear and logarithmic convergence, SIAM J. Numer. Anal. 16, 223 - 240 (1979).

[121] Smith, D. A. and Ford, W. F., Numerical comparisons of nonlinear convergence accelerators, Math. Comput. 38, 481 - 499 (1982).

[122] Steinborn, E. O. and Weniger, E. J., Sequence transformations for the efficient evaluation of infinite series representations of some molecular integrals with exponentially decaying basis functions, J. Mol. Struct. (Theochem) 210, 71 - 78 (1990).

[123] Sternin, B. Y. and Shatalov, V. E., Borel-Laplace Transform and Asymptotic Theory (CRC Press, Boca Raton, 1996).

[124] Suslov, I. M., Divergent perturbation series, J. Exp. Theor. Phys. (JETP) 100, 1188 1234 (2005). 
[125] Szász, O. and Yeardley, N., The representation of an analytic function by general Laguerre series, Pac. J. Math. 8, 621 - 633 (1958).

[126] Szegö, G., Orthogonal Polynomials (American Mathematical Society, Providence, Rhode Island, 1967).

[127] Temme, N. M., Large parameter cases of the Gauss hypergeometric function, J. Comput. Appl. Math. 153, 441 - 462 (2003).

[128] Temme, N. M., Numerical aspects of special functions, Acta Numer. 16, 379 - 478 (2007).

[129] Toeplitz, O., Über allgemeine lineare Mittelbildung, Prace Mat.-Fiz. 22, 113 - 119 (1911).

[130] Tricomi, F. G., Vorlesungen über Orthogonalreihen (Springer-Verlag, Berlin, 1970), 2nd edition.

[131] Tricomi, F. G. and Erdélyi, A., The asymptotic expansion of a ratio of gamma functions, Pacif. J. Math. 1, 133 - 142 (1951).

[132] Trivedi, H. P. and Steinborn, E. O., Numerical properties of a new translation formula for exponential-type functions and its application to one-electron multicenter integrals, Phys. Rev. A 25, $113-127$ (1982).

[133] Villani, M., A summation method for perturbation series with divergent terms, in Bessis, D. (editor), Cargèse Lectures in Physics, volume 5, 461 - 474 (Gordon and Breach, New York, 1972).

[134] Walz, G., Asymptotics and Extrapolation (Akademie Verlag, Berlin, 1996).

[135] Weniger, E. J., Weakly convergent expansions of a plane wave and their use in Fourier integrals, J. Math. Phys. 26, 276 - 291 (1985).

[136] Weniger, E. J., Nonlinear sequence transformations for the acceleration of convergence and the summation of divergent series, Comput. Phys. Rep. 10, 189 - 371 (1989). Los Alamos Preprint math-ph/0306302(http: //arXiv .org).

[137] Weniger, E. J., On the summation of some divergent hypergeometric series and related perturbation expansions, J. Comput. Appl. Math. 32, 291 - 300 (1990).

[138] Weniger, E. J., Interpolation between sequence transformations, Numer. Algor. 3, 477 $-486(1992)$.

[139] Weniger, E. J., On the efficiency of linear but nonregular sequence transformations, in Cuyt, A. (editor), Nonlinear Numerical Methods and Rational Approximation II, 269 282 (Kluwer, Dordrecht, 1994).

[140] Weniger, E. J., Verallgemeinerte Summationsprozesse als numerische Hilfsmittel für quantenmechanische und quantenchemische Rechnungen, Habilitation thesis, Fachbereich Chemie und Pharmazie, Universität Regensburg (1994). Los Alamos Preprint math-ph/0306048 (http: //arXiv . org). 
[141] Weniger, E. J., Nonlinear sequence transformations: A computational tool for quantum mechanical and quantum chemical calculations, Int. J. Quantum Chem. 57, 265 - 280 (1996). Erratum, Int. J. Quantum Chem. 58, 319 - 321 (1996).

[142] Weniger, E. J., A convergent renormalized strong coupling perturbation expansion for the ground state energy of the quartic, sextic, and octic anharmonic oscillator, Ann. Phys. (NY) 246, 133 - 165 (1996).

[143] Weniger, E. J., Computation of the Whittaker function of the second kind by summing its divergent asymptotic series with the help of nonlinear sequence transformations, Comput. Phys. 10, 496 - 503 (1996).

[144] Weniger, E. J., Construction of the strong coupling expansion for the ground state energy of the quartic, sextic and octic anharmonic oscillator via a renormalized strong coupling expansion, Phys. Rev. Lett. 77, 2859 - 2862 (1996).

[145] Weniger, E. J., Performance of superconvergent perturbation theory, Phys. Rev. A 56, 5165 - 5168 (1997).

[146] Weniger, E. J., Prediction properties of Aitken's iterated $\Delta^{2}$ process, of Wynn's epsilon algorithm, and of Brezinski's iterated theta algorithm, J. Comput. Appl. Math. 122, 329 - 356 (2000). Reprinted in Brezinski, C. (editor), Numerical Analysis 2000, Vol. 2: Interpolation and Extrapolation, 329 - 356 (Elsevier, Amsterdam, 2000).

[147] Weniger, E. J., Irregular input data in convergence acceleration and summation processes: General considerations and some special Gaussian hypergeometric series as model problems, Comput. Phys. Commun. 133, 202 - 228 (2001).

[148] Weniger, E. J., A rational approximant for the digamma function, Numer. Algor. 33, $499-507$ (2003).

[149] Weniger, E. J., Mathematical properties of a new Levin-type sequence transformation introduced by Č́̌žek, Zamastil, and Skála. I. Algebraic theory, J. Math. Phys. 45, 1209 - 1246 (2004).

[150] Weniger, E. J., Asymptotic approximations to truncation errors of series representations for special functions, in Iske, A. and Levesley, J. (editors), Algorithms for Approximation, 331 - 348 (Springer-Verlag, Berlin, 2007).

[151] Weniger, E. J., Extended Comment on "One-Range Addition Theorems for Coulomb Interaction Potential and Its Derivatives" by I. I. Guseinov (Chem. Phys. Vol. 309 (2005), pp. 209 - 213), Los Alamos Preprint arXiv:0704.1088v3 [math-ph] (http://arXiv.org) (2007).

[152] Weniger, E. J., Reply to "Extended Rejoinder to "Extended Comment on "One-Range Addition Theorems for Coulomb Interaction Potential and Its Derivatives" by I. I. Guseinov (Chem. Phys., Vol. 309 (2005), pp. 209-213)”, arXiv:0706.0975v2”, Los Alamos Preprint arXiv:0707.3361v1 [math-ph] (http://arXiv.org) (2007).

[153] Weniger, E. J., Further discussion of sequence transformation methods, Subtopic "Related Resources" (R1) on the Numerical Recipes (Third Edition) Webnotes page http: //www.nr.com/webnotes/ (2007). 
[154] Weniger, E. J., One-range and two-range addition theorems, Topical review for Journal of Physics A, in preparation (2008).

[155] Weniger, E. J. and Č́̌žek, J., Rational approximations for the modified Bessel function of the second kind, Comput. Phys. Commun. 59, 471 - 493 (1990).

[156] Weniger, E. J., Čížek, J., and Vinette, F., Very accurate summation for the infinite coupling limit of the perturbation series expansions of anharmonic oscillators, Phys. Lett. A 156, 169 - 174 (1991).

[157] Weniger, E. J., Č́́žek, J., and Vinette, F., The summation of the ordinary and renormalized perturbation series for the ground state energy of the quartic, sextic, and octic anharmonic oscillators using nonlinear sequence transformations, J. Math. Phys. 34, 571 - 609 (1993).

[158] Weniger, E. J., Grotendorst, J., and Steinborn, E. O., Some applications of nonlinear convergence accelerators, Int. J. Quantum Chem. Symp. 19, 181 - 191 (1986).

[159] Weniger, E. J. and Kirtman, B., Extrapolation methods for improving the convergence of oligomer calculations to the infinite chain limit of quasi-onedimensional stereoregular polymers, Comput. Math. Applic. 45, 189 - 215 (2003).

[160] Weniger, E. J. and Liegener, C., Extrapolation of finite cluster and crystal-orbital calculations on trans-polyacetylene, Int. J. Quantum Chem. 38, 55 - 74 (1990).

[161] Weniger, E. J. and Steinborn, E. O., Overlap integrals of $B$ functions. A numerical study of infinite series representations and integral representations, Theor. Chim. Acta 73, 323 - 336 (1988).

[162] Weniger, E. J. and Steinborn, E. O., Nonlinear sequence transformations for the efficient evaluation of auxiliary functions for GTO molecular integrals, in Defranceschi, M. and Delhalle, J. (editors), Numerical Determination of the Electronic Structure of Atoms, Diatomic and Polyatomic Molecules, 341 - 346 (Kluwer, Dordrecht, 1989).

[163] Wimp, J., Sequence Transformations and Their Applications (Academic Press, New York, 1981).

[164] Wynn, P., On a device for computing the $e_{m}\left(S_{n}\right)$ transformation, Math. Tables Aids Comput. 10, 91 - 96 (1956).

[165] Zayed, A. I., Handbook of Function and Generalized Function Transformations (CRC Press, Boca Raton, 1996).

[166] Zeller, K. and Beekmann, W., Theorie der Limitierungsverfahren (Springer-Verlag, Berlin, 1970). 